\title{
Foxo1 deletion promotes the growth of new lymphatic valves
}

\author{
Joshua P. Scallan, ${ }^{1}$ Luz A. Knauer, ${ }^{1}$ Huayan Hou, ${ }^{1}$ Jorge A. Castorena-Gonzalez, ${ }^{2}$ Michael J. Davis, ${ }^{3}$ and Ying Yang ${ }^{1}$ \\ 'Department of Molecular Pharmacology and Physiology, Morsani College of Medicine, University of South Florida, Tampa, Florida, USA. ²Department of Pharmacology, School of Medicine, Tulane University, \\ New Orleans, Louisiana, USA. ${ }^{3}$ Department of Medical Pharmacology and Physiology, University of Missouri, Columbia, Missouri, USA.
}

\begin{abstract}
Patients with congenital lymphedema suffer from tissue swelling in part due to mutations in genes regulating lymphatic valve development. Lymphatic valve leaflets grow and are maintained throughout life in response to oscillatory shear stress (OSS), which regulates gene transcription in lymphatic endothelial cells (LECs). Here, we identified the first transcription factor, Foxo1, that repressed lymphatic valve formation by inhibiting the expression of valve-forming genes. We showed that both embryonic and postnatal ablation of Foxo1 in LECs induced additional valve formation in postnatal and adult mice in multiple tissues. Our quantitative analyses revealed that after deletion, the total number of valves in the mesentery was significantly $(P<0.01)$ increased in the Foxo $1^{L E C-K O}$ mice compared with Foxo ${ }^{f l / f l}$ controls. In addition, our quantitative real-time PCR (RT-PCR) data from cultured LECs showed that many valve-forming genes were significantly ( $P$ $<0.01)$ upregulated upon knockdown of FOX01. To confirm our findings in vivo, rescue experiments showed that Foxc2 ${ }^{+/-}$ mice, a model of lymphedema-distichiasis, had $\mathbf{5 0 \%}$ fewer lymphatic valves and that the remaining valves exhibited backleak. Both valve number and function were completely restored to control levels upon Foxo1 deletion. These findings established FOXO1 as a clinically relevant target to stimulate de novo lymphatic valve formation and rescue defective valves in congenital lymphedema.
\end{abstract}

\section{Introduction}

The lymphatic vasculature plays an essential role in maintaining fluid homeostasis in the body. The lymphatic capillaries absorb interstitial fluid to form lymph and the collecting lymphatic vessels return lymph to the venous vasculature (1-4). To maintain forward lymph flow against the higher hydrostatic pressure in the veins, the collecting vessels grow bicuspid intraluminal valves. The lymphatic valves are comprised of 2 leaflets and each leaflet has a layer of lymphatic endothelial cells (LECs) on each side with an extracellular matrix core sandwiched between them $(1,2)$. The 2 leaflets open and close in a pressure-dependent manner to allow lymph to move forward or to prevent backflow into the tissues $(5,6)$.

Defective valves are strongly associated with the human disease lymphedema, which features severely swollen tissues caused by interstitial fluid accumulation associated with adipose tissue deposition and fibrosis (7-9). Lymphedema occurs due to genetic mutations and/or lymph node dissection during cancer treatment. There are several correlations between defective valves and lymphedema: (a) many gene mutations that cause human congenital lymphedema or lymphatic abnormalities have valve defects in the corresponding mouse models (e.g., FOXC2, RASA1, ITGA9, GATA2, EPHB4, CX43, PIEZO1; refs. 10-26); (b) dermal backflow is consistently observed in patients with lymphedema,

Conflict of interest: The authors have declared that no conflict of interest exists. Copyright: (ㄷ 2021, American Society for Clinical Investigation.

Submitted: July 16, 2020; Accepted: June 3, 2021; Published: July 15, 2021

Reference information: / Clin Invest. 2021;131(14):e142341.

https://doi.org/10.1172/JCl142341. which can only occur with defective valves $(27,28)$; (c) patients with breast cancer who have point mutations in genes that regulate valve development (e.g., FOXC2) are at a higher risk of developing lymphedema after lymph node removal (29); and (d) stagnant lymph flow initiates lymphedema (30-35). However, little is known about how to artificially induce valve formation or prevent valve regression.

Previously, we showed that VE-cadherin signaling is required for lymphatic valve maintenance by activating the $\beta$-catenin and AKT signaling pathways (36). Although the downstream targets of AKT signaling in the lymphatic vasculature have not been completely identified, AKT phosphorylation is known to inactivate FOXO1 by excluding it from the nucleus $(37,38)$. FOXO1 belongs to the FOXO subfamily of forkhead transcription factors, which are evolutionarily conserved and unified by the presence of a highly homologous DNA-binding domain termed the forkhead box (39). Foxo1 is abundantly expressed in blood endothelial cells (BECs) and plays a major role in regulating blood vessel growth. Global Foxo1 knockout animals die around embryonic (E) day 10.5 with abnormal vascular remodeling $(40,41)$. Moreover, endothelial cell-specific knockout of Foxo1 results in embryonic lethality at E10.5 with overgrowth of blood vessels caused by increased proliferation in BECs (42), which indicates that FOXO1 acts as a negative regulator during vascular development. Here, we hypothesized that FOXO1 regulates lymphatic valve maintenance downstream of VE-cadherin. Unexpectedly, lymphatic-specific deletion of FOXO1 led to the development of markedly more valves in the mesentery than in littermate controls by activating the expression of several shear stress-sensitive genes involved in valve develop- 

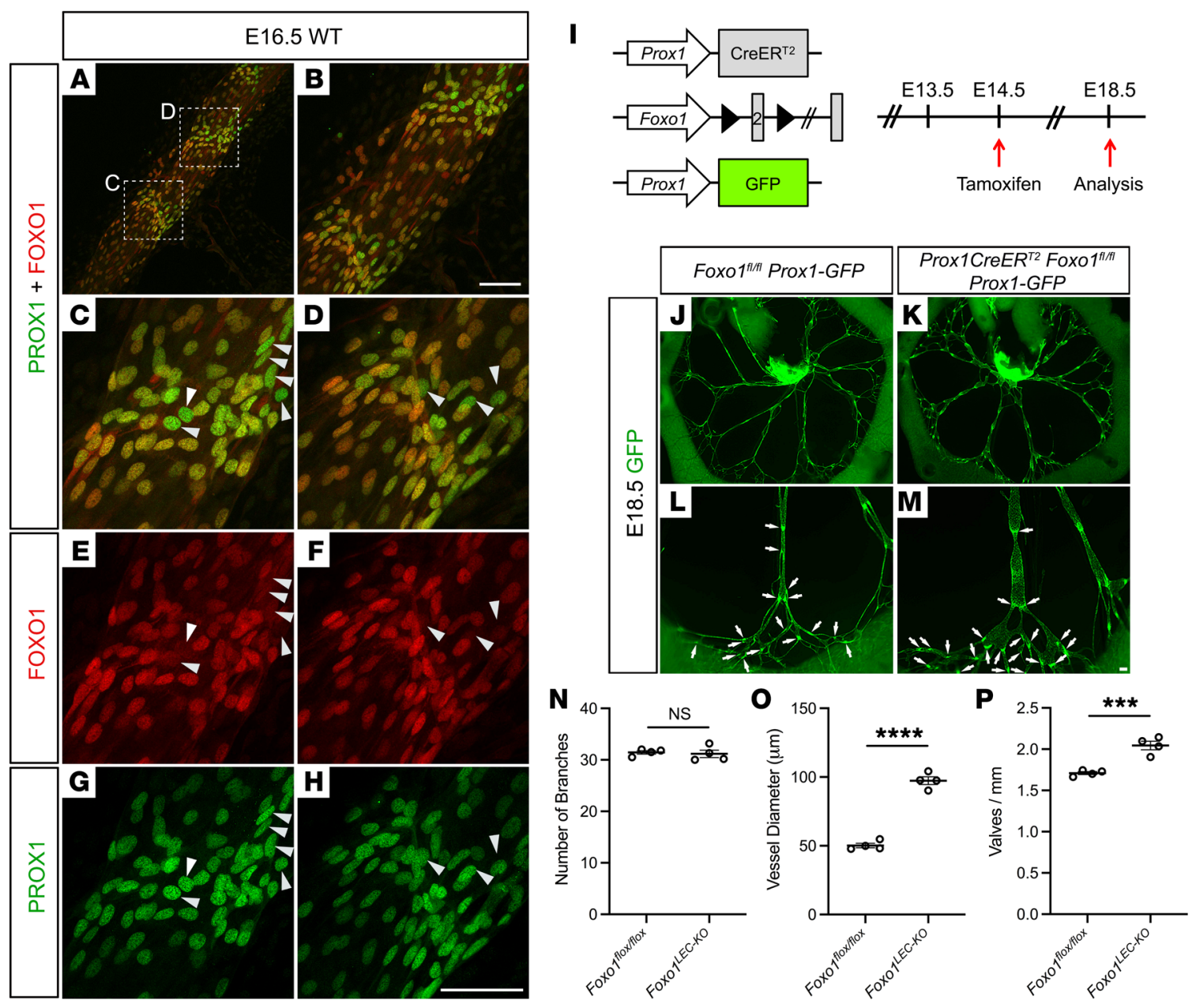

Figure 1. Loss of FOXO1 nuclear localization during valve specification and deletion of Foxo1 in the lymphatic vasculature results in increased valve-forming areas. (A-H) Whole-mount immunostaining of PROX1 (green), FOXO1 (red), and DAPI (blue) in WT E16.5 mesentery. Panels C and D are the higher magnification images of the white squared areas in $\mathbf{A}$, indicating the valve-forming areas. FOXO1 nuclear localization is missing in many PROX1-positive cells in the valve-forming areas (white arrowheads). (I) Tamoxifen injection procedure for embryonic deletion of Foxo1. (J-P) Fluorescence imaging of the morphology of the mesenteric lymphatic vasculature indicated by GFP expression from E18.5. The GFPhi valves are indicated by white arrows. (N) The average number of the branches located in the thinner precollecting lymphatic vessels close to the intestinal wall from 10 large collecting vessels in each mesentery. $(\mathbf{O})$ The average diameter of the large collecting vessels from each mesentery. $(\mathbf{P})$ Valves per millimeter from each mesentery. Four controls and 4 knockout mesenteries were used in each analysis. All values are mean \pm SEM. ${ }^{* * *} P=0.0002$, ${ }^{* * * *} P<0.0001$, calculated by unpaired Student's $t$ test. Scale bars are $50 \mu \mathrm{m}$ in $\mathbf{B}$ and $\mathbf{H}$, and $100 \mu \mathrm{m}$ in $\mathbf{M}$.

ment. Furthermore, we showed that deletion of Foxo1 in a mouse model of human disease lymphedema-distichiasis syndrome can fully restore the valve defects in these animals.

\section{Results}

Nuclear FOXO1 expression is lost during lymphatic valve specification. Lymphatic valves are crucial for maintaining the forward flow of lymph against a hydrostatic pressure gradient experienced by collecting lymphatic vessels. Foxo1 is a transcription factor that typically acts as a repressor of gene expression in vascular endothelium (42). While it has been reported that endothelial deletion of Foxo1 increases BEC and LEC proliferation (42-44), a functional role for Foxo1 in lymphatic valves has not been reported. Since the expression pattern of FOXO1 in LECs is unknown during valve development, we performed wholemount immunostaining (Figure 1, A-H) of FOXO1 and the LEC marker PROX1 in the mesentery, a valve-enriched tissue (45, 46). In WT embryos at E16.5, LECs become specified for valve formation by upregulating PROX1 $(2,45)$. While we found that FOXO1 colocalized with PROX1 in the nuclei of non-valve LECs (Figure 1B), many LECs in the valve specification area (Figure 1, $\mathrm{C}$ and D) lacked nuclear expression of FOXO1 and exclusively expressed a high level of PROX1 (Figure 1, $\mathrm{C}-\mathrm{H}$, arrowheads). The nuclear localization of FOXO1 is dynamically regulated by AKT. FOXO1 phosphorylation by AKT excludes it from the nucleus, thereby inhibiting FOXO1 activity $(37,38)$. Therefore, our data indicate that the activity of FOXO1 is decreased in the LECs destined to become valve leaflets. Moreover, in our previous publication, we showed that direct activation of AKT induced additional lymphatic valve formation in WT lymphatic vessels (36). Together, these data suggested that FOXO1 may act as a repressor during lymphatic valve formation. 
A
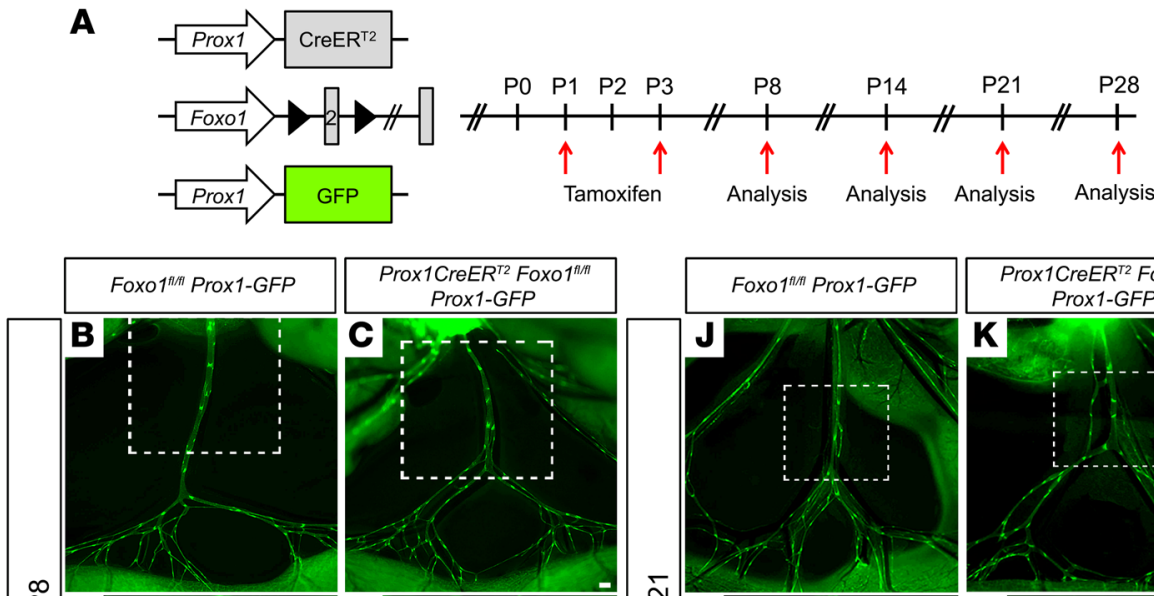

$\stackrel{\infty}{\circ}$
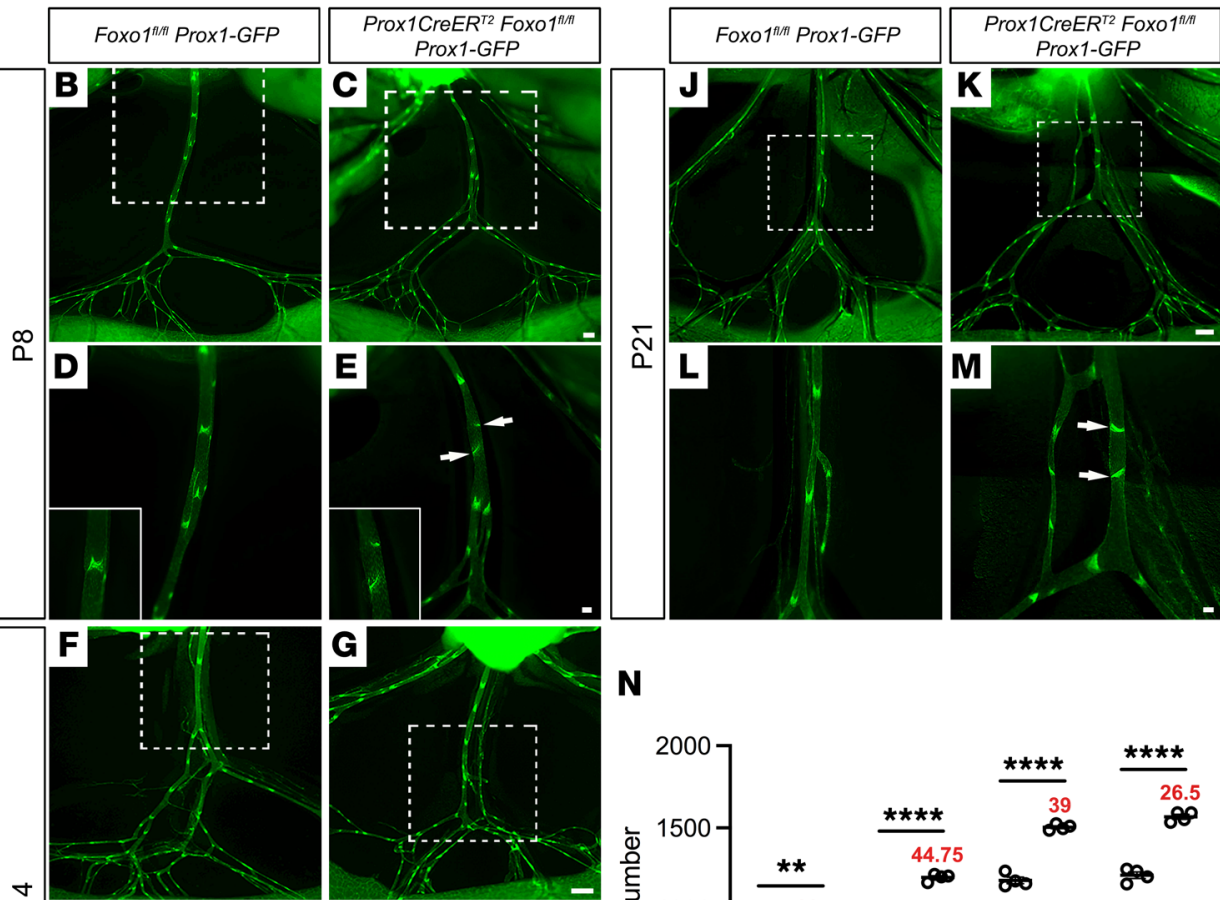

$\overline{\mathrm{C}} \mathbf{H}$

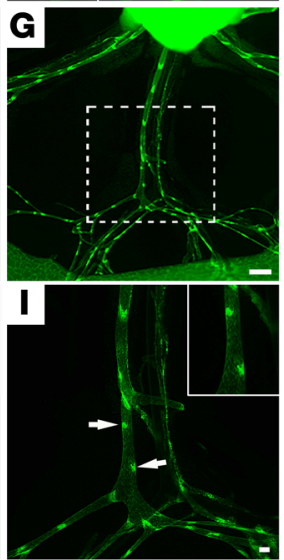

$\mathbf{N}$
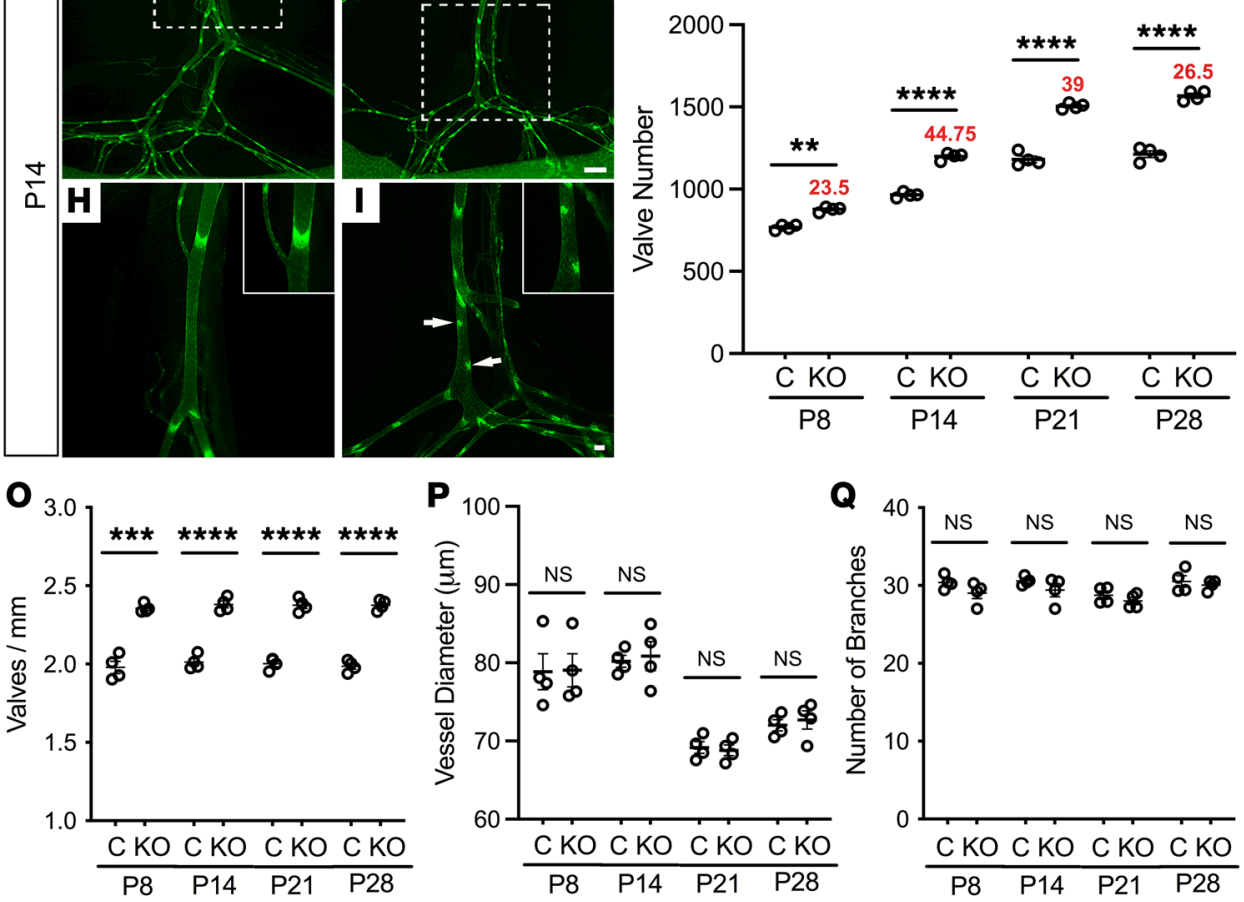

Figure 2. Postnatal deletion of Foxo1 in the lymphatic vasculature leads to additional valve growth. (A) Tamoxifen injection procedure for postnatal deletion of Foxo1. (B-M) Fluorescence imaging of the morphology of the mesenteric lymphatic vasculature indicated by GFP expression from P8, P14, and P21. The Foxo1 ${ }^{\mathrm{LEC}-K O}$ mesentery has many valve initiation and elongation areas (white arrows). D, E, H, $\mathbf{I}, \mathbf{L}$, and $\mathbf{M}$ are the higher magnification images of the white squared areas in B, C, F, G, J, and K. (N) Total number of mature valves from each mesentery. The red numbers are the average number of immature valves. (0) Valves per millimeter from each mesentery. (P) The average diameter of the large collecting vessels from each mesentery. (Q) The average number of the branches located in the thinner precollecting lymphatic vessels close to the intestinal wall from 10 large collecting vessels in each mesentery. Scale bars are $500 \mu \mathrm{m}$ in $\mathbf{C}, \mathbf{G}$, and $\mathbf{K}$, and $100 \mu \mathrm{m}$ in $\mathbf{E}, \mathbf{I}$, and $\mathbf{M}$. All values are mean \pm SEM. Four controls and 4 knockout mesenteries were used in each analysis $(\mathbf{N}-\mathbf{Q})$. ${ }^{* *} P<0.01,{ }^{* * *} P<0.001$ ${ }^{* * * *} P<0.0001$, calculated by unpaired Student's $t$ test.
Embryonic deletion of Foxo1 enhances valve specification. To investigate the function of Foxo1 in lymphatic valve formation, we used a tamoxifen-inducible (TM-inducible) LEC-specific Cre line (47), Prox1CreER ${ }^{T 2}$, to conditionally delete Foxo1 (Foxo1 ${ }^{f / f}$; ref. 48) from lymphatic, but not blood, endothelium. To enable visualization of the lymphatic vasculature in vivo, we also bred an LEC reporter line (49), Prox1-GFP, with the LEC-specific Foxo1 knockout mice to yield Prox1CreER $R^{T 2}$ Foxo1 ${ }^{f l / f l}$ Prox1-GFP mice (hereafter referred to as Foxo1 $\left.{ }^{L E C-K O}\right)$. Controls were littermates that lacked the CreER ${ }^{\mathrm{T} 2}$ allele (i.e., Foxo1 ${ }^{\ell / f l}$ Prox1-GFP) but were treated with the same dose of TM (Figure 1I). We administered TM on E14.5, the beginning of the formation of collecting lymphatic vessels in the mesentery (50). Administration of TM led to approximately $100 \%$ deletion of Foxo1 in LEC by E18.5 (Supplemental Figure 1, A-D; supplemental material available online with this article; https://doi.org/10.1172/JCI142341DS1). Although the number of lymphatic vessel branches in the Foxo1 ${ }^{L E C-K O}$ mesenteries was similar to the controls and no extra lymphangiogenesis or sprouting was observed (Figure 1, J-N), the collecting lymphatic vessels in the E18.5 Foxo1 ${ }^{L E C-K O}$ were dilated compared with controls (Figure 


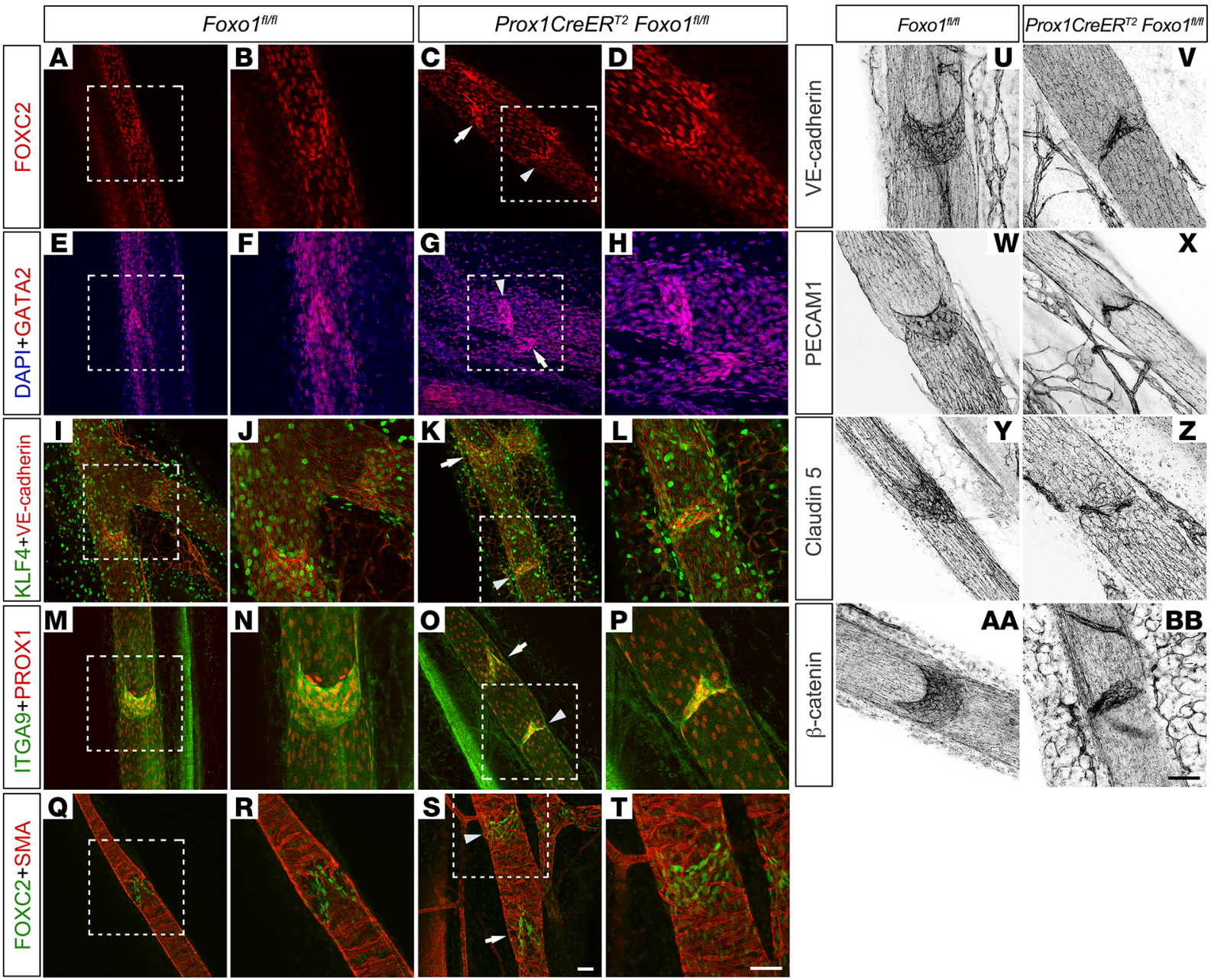

Figure 3. Loss of Foxo1 in the LECs leads to the formation of new valves that express high levels of FOXC2, PROX1, GATA2, KLF4, and ITCA9. (A-BB) Whole-mount immunostaining of mesenteries collected from P10 (TM injected at P1/P3) control and Foxo1LEC-Ko with FOXC2 (red) (A-D); 2-(4-amidinophenyl)-1H-indole-6-carboxamidine (DAPI; blue) and GATA2 (red) (E-H); KLF4 (green) and VE-cadherin (red) (I-L); ITCA9 (green) and PROX1 (red) (M-P); FOXC2 (green) and SMA (red) (Q-T); VE-cadherin (U-V); PECAM1 ( $\mathbf{W}$ and $\mathbf{X}$ ); Claudin 5 (Y and Z); $\beta$-catenin (AA and BB). B, D, F, H, J, L, N, P, R, and T are the higher magnification images of the white squared areas in $\mathbf{A}, \mathbf{C}, \mathbf{E}, \mathbf{C}, \mathbf{I}, \mathbf{K}, \mathbf{M}, \mathbf{O}, \mathbf{Q}$, and $\mathbf{S}$. Scale bars are $50 \mu \mathrm{m}$. The white arrows indicate the mature valves and the white arrowheads indicate the immature valves. Ten valves per mesentery from 3 controls and 3 knockout mesenteries were used in the analysis.

10), indicating that Foxo1 controls the size of the vessels during

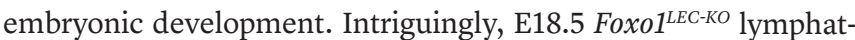
ic vessels contained many PROX $1^{\text {hi }}$ valves (Figure $1 \mathrm{~K}$, arrows). Quantitative analysis showed that the Foxo1 ${ }^{\text {LEC-KO }}$ lymphatics had significantly $(P=0.0009)$ more valves than the controls (Figure 1P), indicating that valve formation was more active in the Foxo1 ${ }^{L E C-K O}$ animals. Together, this demonstrated that loss of Foxo1 enhanced lymphatic valve development, indicating a role for Foxo1 as a repressor of the valve gene program.

Postnatal deletion of Foxo1 leads to enhanced lymphatic valve formation. To further analyze the function of Foxo1 in lymphatic valve formation, we deleted Foxo1 postnatally to gain better temporal resolution for distinguishing the different stages of valve development. We administered TM at postnatal day (P) 1 and P3 (P1/P3) and analyzed both the mesenteric and axillary lymphatic vessels in the Foxo1 ${ }^{L E C-K O}$ and controls at P8, P14, P21, and P28 (Figure 2, $\mathrm{A}-\mathrm{M})$. We verified the deletion was approximately $100 \%$ efficient using whole-mount immunostaining (Supplemental Figure 1, E-H).
Foxo1 ${ }^{\text {LEC-KO }}$ and control mice were grossly similar in terms of weight and appearance at all 4 time points (data not shown). At P8, P14, and P21, the mesenteric lymphatic vessels from the Foxo ${ }^{1 E C-K O}$ mice displayed many $\mathrm{PROX}^{\mathrm{hi}}$ valve-forming areas (Figure 2E, I, and M, arrows) (45). Following a previous staging system for lymphatic valves (45), some of these areas were in the valve initiation stage while others formed ring-like structures (45). This was confirmed in P8 axillary lymphatic vessels (Supplemental Figure 2). Two possibilities could explain this observation: (a) these valves were under regression because regressing valves slowly lose leaflets $(36,51)$, or (b) the vessels were actively forming new valves. To determine which possibility explained the phenotype of the Foxo $1^{L E C-K O}$ mice, we quantified the total number of valves in the Foxo $1^{\text {LEC-KO }}$ and the control mice. If the valves were regressing, then the total number of valves in the Foxo ${ }^{1 E C-K O}$ mice would be decreased compared with controls. However, if more valves were actively forming, then the Foxo ${ }^{\text {LEC-KO }}$ mice would have more valves than controls. We counted the total number of mature, fully formed valves (valves with 2 leaf- 

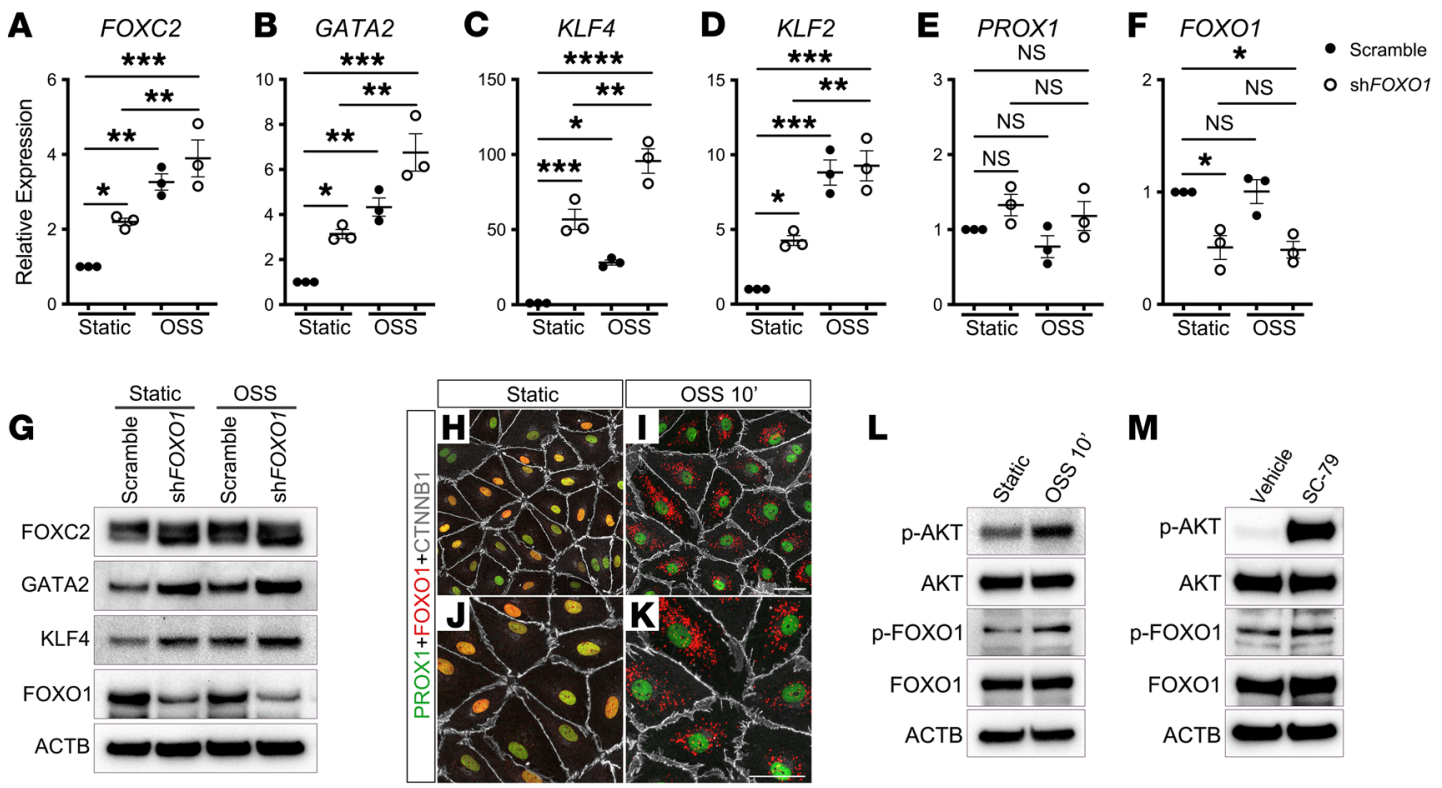

$\mathbf{N}$

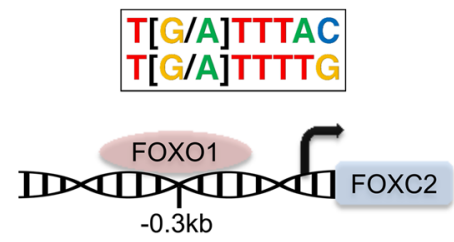

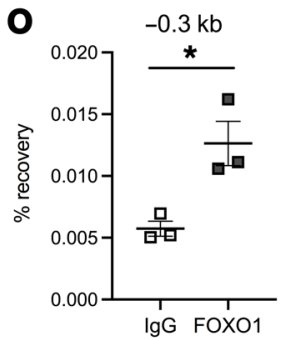

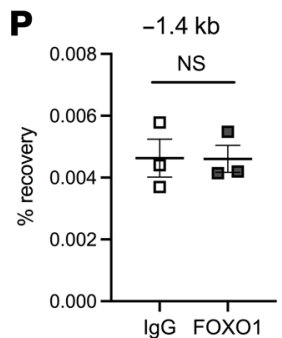

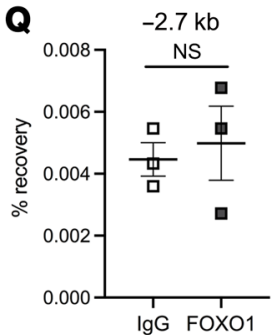

Figure 4. Loss of Foxo1 upregulates the expression levels of valve-forming genes in postnatal vessels. (A-F) qRT-PCR was performed for the indicated genes using hdLECs cultured under static or OSS conditions, transfected with a control scramble (closed circles) or shRNA against FOXO1 (open circles). (C) Western blot was performed for the indicated genes using hdLECs cultured under static or OSS conditions, transfected with a control scramble or shRNA against FOX01. (H-K) Immunostaining was performed for PROX1 (green), FOXO1 (red), and CTNNB1 (white) using hdLECs cultured under static or OSS conditions. Scale bars are $50 \mu \mathrm{m}$. (L) Western blot was performed for the indicated genes using hdLECs cultured under static or OSS conditions. (M) Western blot was performed for the indicated genes using hdLECs cultured under vehicle or SC-79 treatment. (N) FOXO1 binding site on the promoter area of FOXC2 gene. (0-Q) ChIP assay was performed using IgG or an antibody specific to FOXO1. QRT-PCR was performed using primers flanking the FOXO1-binding site in the $-0.3 \mathrm{~kb}$ location. As negative controls, primers flanking more upstream locations $(-1.4 \mathrm{~kb}$ and $-2.7 \mathrm{~kb})$ were used. All values are mean $\pm \mathrm{SEM}$ of $n=3$ experiments. Two-way ANOVA was performed with Tukey's multiple comparisons in A-F $\left({ }^{*} P<0.05,{ }^{* *} P<0.01,{ }^{* * *} P<0.001,{ }^{* * * *} P<0.0001\right)$. ${ }^{*} P<0.05$ and NS were calculated by unpaired Student's $t$ test in $\mathbf{0}-\mathbf{Q}$. Scale bars are $50 \mu \mathrm{m}$ in I and $\mathbf{K}$.

lets indicated in Figure 2, D and $\mathrm{H})$ in the mesentery at all 4 stages. Foxo1 ${ }^{L E C-K O}$ mice had significantly $(P<0.01)$ increased numbers of lymphatic valves with complete valve leaflets at P8, P14, P21, and P28 (Figure $2 \mathrm{~N}$ ). The total number of mature lymphatic valves at P8, P14, P21, and P28 in the Foxo1 ${ }^{\text {LEC-KO }}$ mesenteries was $14 \%$, $24 \%, 27 \%$, and $26 \%$ higher than the controls, respectively. These findings confirmed that this enhancement in lymphatic valve formation is persistent during growth. To test whether the immature valve-forming spots matured into fully formed valves, we counted the number of valve-forming areas indicated in Figure 2, E, I, and M at all 4 stages. The average number of immature valves at P8, P14, P21, and P28 were 23.5, 44.75, 39, and 26.5, respectively (Figure $2 \mathrm{~N}$, red numbers). The decreased number of immature valves from P21 to P28 combined with the consistently increased total number of mature valves at these 2 stages indicated that many of the valve-forming spots had developed into mature valves over time. To further confirm that valve formation was induced by inactivation of Foxo1, we normalized valve number to total length (milli- meter) of each collecting lymphatic vessel to obtain the valve number per millimeter in both Foxo1 ${ }^{L E C-K O}$ and controls. The Foxo1 ${ }^{L E C-K O}$ vessels displayed a significantly $(P<0.001)$ higher valve number per millimeter than the controls (Figure 2O), which indicated that the distance between each valve was shorter in the Foxo ${ }^{\text {LEC-KO }}$ than the controls. Meanwhile, unlike the embryos, postnatal deletion of Foxo1 did not cause vessel dilation in the mesentery (Figure 2P). Since enhanced branching would increase the surface area for valves to form, we counted the number of branch points. There was no significant difference in the average number of branches between the Foxo1 ${ }^{\mathrm{LEC}-K O}$ and controls (Figure 2Q). Likewise, the Foxo1 ${ }^{L E C-K O}$ axillary lymphatic vessels that connect the inguinal and axillary lymph nodes exhibited many valves and valve-forming sites (Supplemental Figure 2E, arrows and arrowheads). The number of valves per millimeter was significantly $(P=0.0088)$ higher in the Foxo1 ${ }^{L E C-K O}$ axillary vessels compared with the controls at P8, and the diameter of the axillary vessels did not change upon Foxo1 deletion (Supplemental Figure 2, F and G). Together, these data 
A
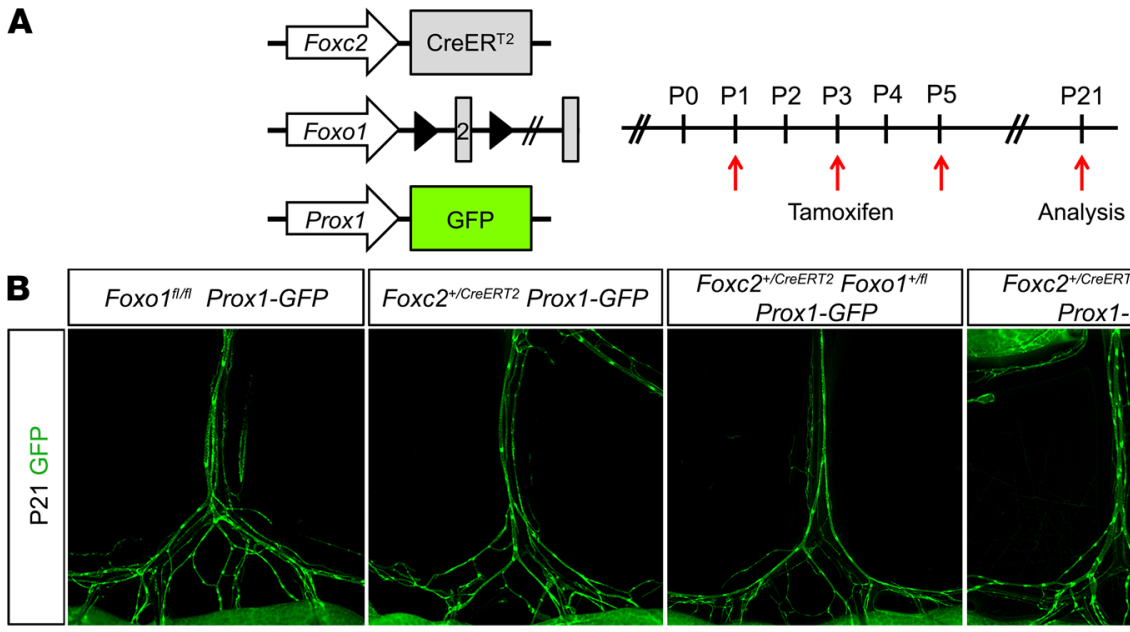

Tamoxifen

Analysis
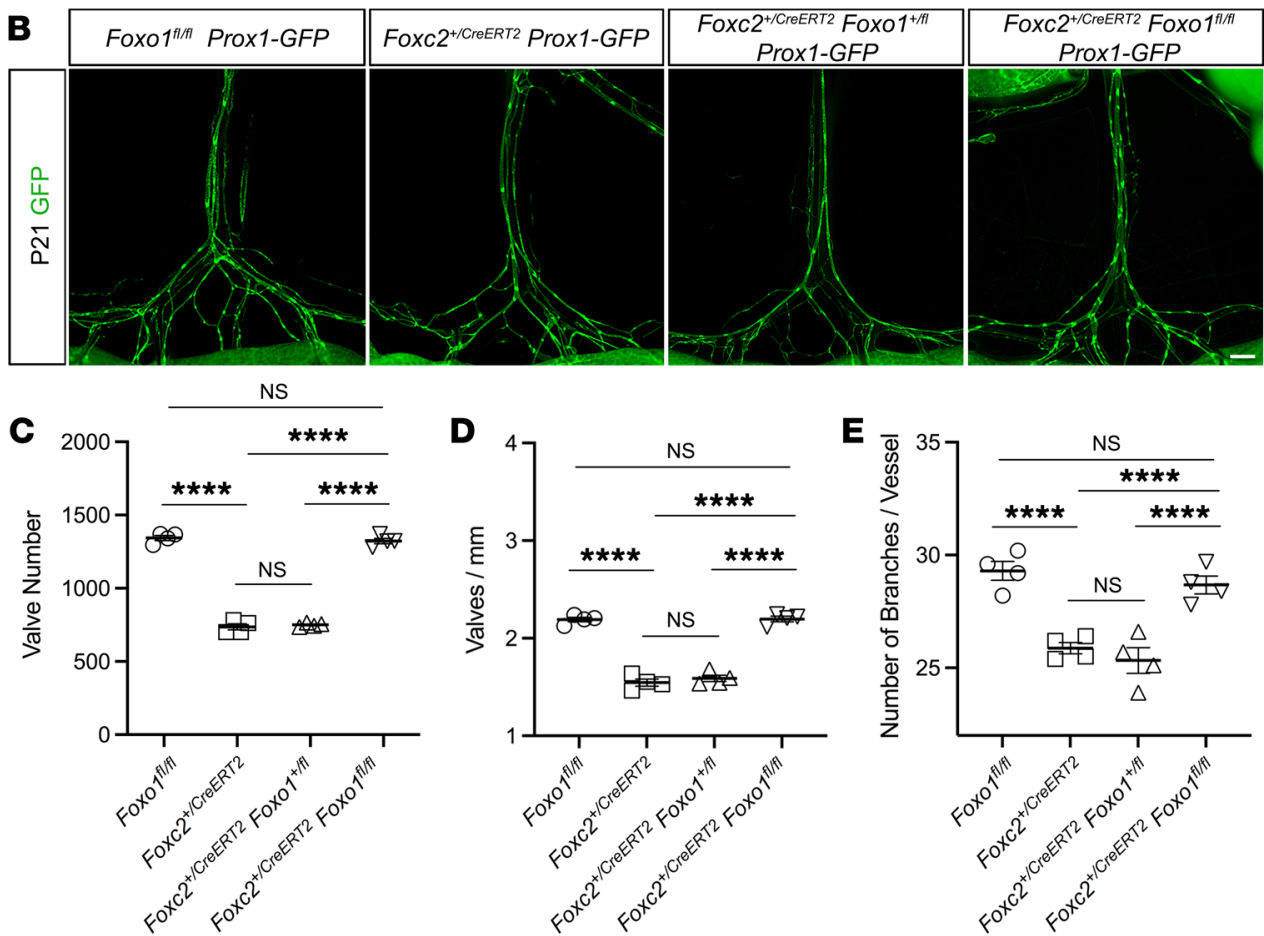

E

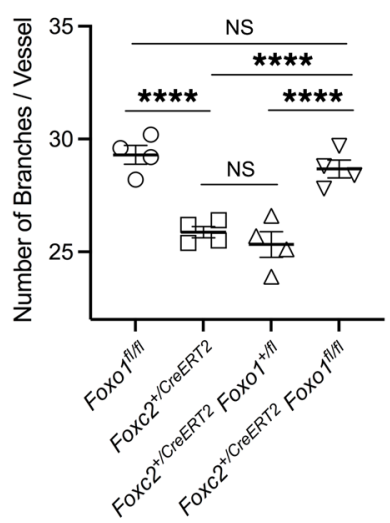

Figure 5. Loss of Foxo1 restores the number of valves and the number of branches in Foxc2 heterozygous mice. (A) Tamoxifen injection procedure for postnatal deletion of Foxo1 in the Foxc2 heterozygous background. (B) Fluorescence imaging of the morphology of the mesenteric lymphatic vasculature indicated by GFP expression from P21. (C) Total number of mature valves from each mesentery. (D) Valves per millimeter from each mesentery. (E) The average number of the branches located in the thinner precollecting lymphatic vessels close to the intestinal wall from 10 large collecting vessels in each mesentery. Four controls and 4 knockout mesenteries were used in each analysis. All values are mean \pm SEM. One-way ANOVA was performed with Tukey's multiple comparisons test ${ }^{* *^{* *} P<}$ 0.0001). Scale bar is $500 \mu \mathrm{m}$ in $\mathbf{B}$. showed that deletion of Foxo1 neither increased the vessel diameter nor the number of the branches in both the mesenteric and the axillary vessels, indicating that we induced additional formation of fully formed valves without increasing surface area.

In addition to the mesenteric and the axillary lymphatic vessels, we also analyzed the lymphatics in the ear at P21 following the same TM injection procedure (Supplemental Figure 3A). Unlike the mesentery, the numbers of valves were not significantly different in the ears and the lymphatic vessels were dilated after the loss of Foxo1 (Supplemental Figure 3, B-G). Ki67 staining indicated that cell proliferation was increased in the Foxo1 ${ }^{L E C-K O}$ ears compared with the controls (Supplemental Figure 3, H, I, and L). Most of these dilated lymphatic vessels had reduced smooth muscle cell coverage (Supplemental Figure 3, J, K, and M).

While the data thus far demonstrate that early postnatal deletion of Foxo1 from LECs leads to enhanced valve formation at multiple stages, this is within a period of rapid development where valves are both forming and being maintained. To investigate whether Foxo1 deletion in an adult mouse likewise enhances valve formation, we induced Foxo1 deletion in 7-weekold mice and analyzed the mesenteric lymphatic valves 2 weeks later. Because of abundant adipose tissue covering the vessels, we were unable to accurately distinguish between mature valves and valve-forming areas in the adult mice. Instead, we compared the total number of valves. Consistently, the adult Foxo1 ${ }^{\text {LEC-KO }}$ vessels had significantly $(P=0.0035)$ more valves than the con- trols. To further demonstrate that valve formation was induced by inactivation of Foxo1, we measured and compared the distance between the valves in the segment of the large collecting vessels between the lymph nodes and the first branch in both Foxo1 ${ }^{L E C-K O}$ and controls because it is impossible to measure the length of the thinner branched precollecting vessels closer to the intestinal wall that were behind the adipose tissues. The distance between each valve was shorter in the Foxo1 ${ }^{L E C-K O}$ than in the controls (Supplemental Figure 4). Thus, ablation of Foxo1 augmented lymphatic valve formation not only in the postnatal period, but also in the adult.

Although Prox1CreER $R^{T 2}$ is specific for LECs within the vasculature, Prox 1 is expressed in many other tissues (e.g. brain, liver, eye, skeletal muscle, and pancreas) along with Foxo1, which is similarly expressed in the brain, skeletal muscles, liver, and pancreas (52-55). Although deletion of Foxo1 from other cell types might cause additional phenotypes, the Foxo1 ${ }^{\mathrm{LEC}-K O}$ mice deleted at P1/P3 survived for more than 12 months with no obvious difference from their litter mate controls in terms of weight and life span (data from 5 pairs of control and knockout, not shown). To study an endothelial-specific deletion of Foxo1, we crossed the inducible, pan-endothelial Cre line (56) Cdh5-CreER $R^{T 2}$ with Foxo1 ${ }^{f / f l}$ mice. Lineage tracing indicated that injection of TM at P1, P3, and P5 leads to approximately $100 \%$ efficient deletion using the $\mathrm{R} 26-\mathrm{mT} / \mathrm{mG}$ Cre-reporter mouse (data not shown). To count the number of valves in the mesentery, we again used the Prox1-GFP strain to obtain Cdh5-CreER ${ }^{T 2}$ Foxo $1^{f / f l}$ 

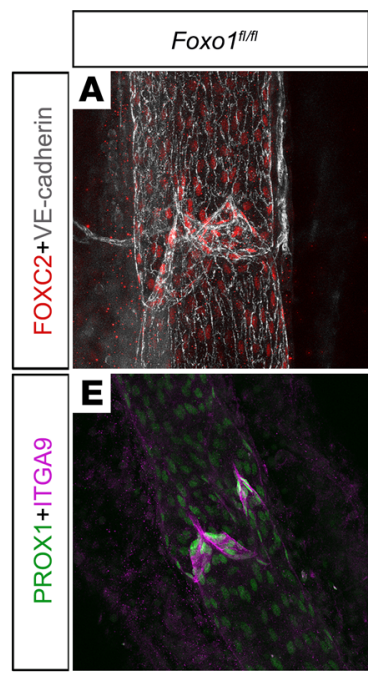
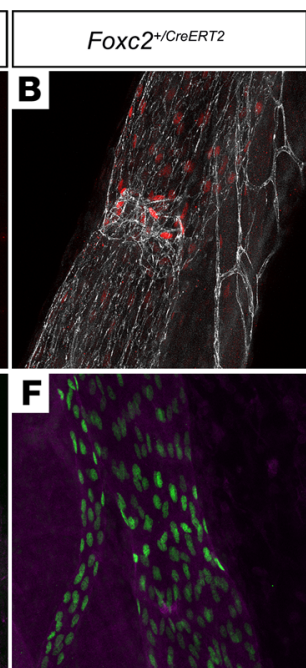
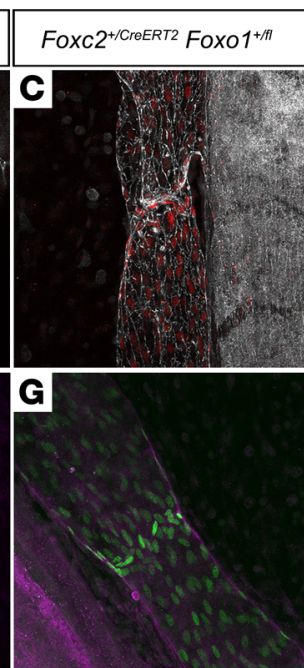

G
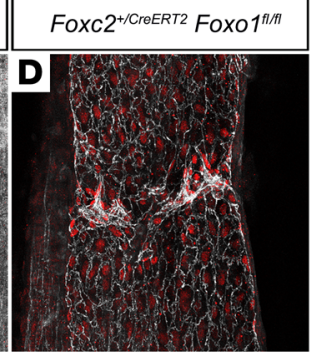

H

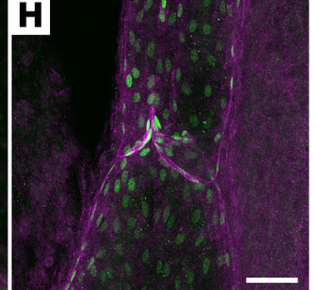

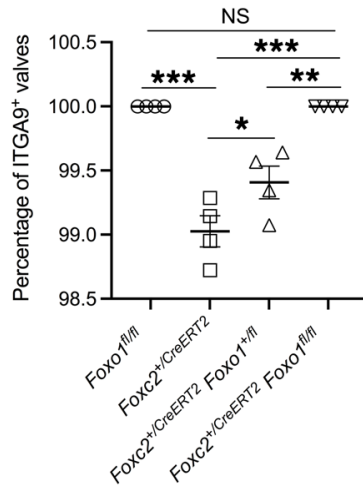

Figure 6. Loss of Foxo1 restores the morphology of valves in Foxc2 heterozygous mice. (A-H) Whole-mount immunostaining of mesenteries collected

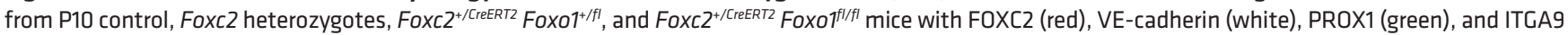
(purple). (I) The percentage of Itga9- positive cells from each mesentery. Scale bar is $50 \mu \mathrm{m}$ in $\mathbf{H}$. Four controls and 4 knockout mesenteries were used in each analysis. All values are mean \pm SEM. One-way ANOVA was performed with Tukey's multiple comparisons test $\left({ }^{*} P<0.05,{ }^{* *} P<0.01,{ }^{* * *} P<0.001\right)$.

Prox1-GFP (Foxo1 $\left.{ }^{E C-K O}\right)$ mice and Foxo1 ${ }^{l / f l}$ Prox1-GFP littermate controls. Similar to the Foxo1 ${ }^{L E C-K O}$ animals, the Foxo1 ${ }^{E C-K O}$ mesentery had many valve-forming areas (Supplemental Figure 5, B-E, arrows). We counted the total number of mature and immature valves (Supplemental Figure $5 \mathrm{~F}$ ) in the mesentery at P14. Foxo1 ${ }^{\text {EC-KO }}$ mice had significantly $(P=0.0012)$ increased numbers of lymphatic valves with complete valve leaflets (Supplemental Figure 5F). These data demonstrate that deletion of Foxo1 from the lymphatic vasculature stimulates new valve growth in a cell-autonomous manner.

In addition to Foxo1, Foxo3 is also expressed in endothelial cells (43). To investigate whether deletion of Foxo3 from LECs leads to new valve formation, we generated Prox1CreERT2 Foxo $3^{f / f l}$ Prox1GFP $\left(\right.$ Foxo $\left.3^{L E C-K O}\right)$ mice and control Foxo $3^{\text {fl/fl }}$ mice. After TM at P1/ P3, the pups were analyzed 2 weeks after deletion at P14 (Supplemental Figure 6A). We imaged the mesentery and counted the total number of valves. The morphologies of the lymphatic vessels and valves in the Foxo ${ }^{L E C-K O}$ mesentery were indistinguishable from controls and the total number of valves were not significantly different in the Foxo $3^{L E C-K O}$ and control animals (Supplemental Figure $6, \mathrm{~B}-\mathrm{F})$. Moreover, to investigate the combinatorial effect of deleting both Foxo1 and Foxo3, we generated Prox1CreERT2 Foxo1 ${ }^{\mathrm{fl} / \mathrm{fl}}$ Foxo3 ${ }^{\text {fl/fl }}$ Prox1-GFP (Foxo1 Foxo3 ${ }^{\text {LEC-KO }}$ ) mice and control Foxo1 Foxo $3^{f / / f l}$ mice. After TM at P1/P3, the pups were analyzed 2 weeks after deletion at P14 (Supplemental Figure 7A). Similar to the Foxo1 single deletion, the mesenteries from Foxo1 and Foxo3 double knockouts exhibited many valve-forming areas (Supplemental Figure 7, B-E, white arrows). More importantly, compared with the Foxo $1^{\text {LEC-KO }}$ single knockouts, the total number of mature valves was not significantly different in the Foxo1/3 $3^{\text {LEC-KO }}$ double knockouts. While Foxo1/3 $3^{\text {LEC-KO }}$ double knockouts had a significantly $(P<0.0001)$ increased total number of mature valves compared with floxed controls, the extent of new valve growth was very similar to the Foxo1 ${ }^{\text {LEC-KO }}$ single knockouts (Supplemental Figure 7F). These data indicate that Foxo3 does not participate in new valve formation.
New valves induced by Foxo1 deletion express valve-specific genes and exhibit normal junctions. To characterize the newly formed valves in the Foxo1 ${ }^{\text {LC-KO }}$ animals, we performed whole-mount immunostaining in the postnatal mesenteries following the same tamoxifen injection protocol as in Figure 2A. Due to the fact that we currently did not have a method to distinguish the newly formed mature valves that were induced by loss of Foxo1 from the existing mature valves based on their morphology, we focused on imaging both the fully formed valves (valves with 2 leaflets) and the incomplete valves that were observed in the Foxo1 ${ }^{L E C-K O}$ mesenteries but rarely seen in the control tissues (Figure 3, A-T). The lymphatic valve cells at E16.5 begin to be specified by an upregulation of a set of valve-forming transcription factors: PROX1, FOXC2, and GATA2 (2). The loss of either Foxc2 or Gata2 leads to a failure in valve formation during embryonic development and failed maintenance of the valve structures after they are formed $(10,15,45,51)$. Although it remains unknown whether Prox1 deletion results in the loss of peripheral valves, Prox1 activity is required for the formation of the lymphovenous valves that connect the lymphatic system and the blood circulation (57). Both mature valves and the valve-form-

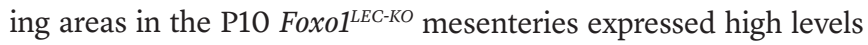
of FOXC2, GATA2, and PROX1 (Figure 3, C, G, and O, arrows and arrowheads), indicating that the normal valve-forming program was active in the absence of Foxol. The staining of Cdh5-CreER ${ }^{T 2}$ Foxo1 $^{f / f l}$ (Supplemental Figure 5, G-N) and Prox1CreERT2 Foxo1 $1^{f / f l}$ Foxo $3^{\text {fl/fl }}$ vessels (Supplemental Figure 7, K-R) displayed similar expression patterns of FOXC2 and PROX1. To confirm that deletion of Foxo1 activated the expression of Foxc2, Gata2, and Prox1 through fluid shear stress (FSS) mechanisms, we analyzed the expression of the FSS responsive genes Foxc2, Gata2, and Klf4, because FSS is required for lymphatic valve formation and the expression of these transcription factors in LECs is known to be increased by oscillatory FSS $(45,58,59)$. While KLF4 was expressed in many types of cells besides LECs in the mesentery, both the mature valves and the 
A

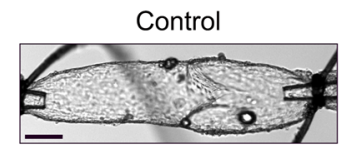

B

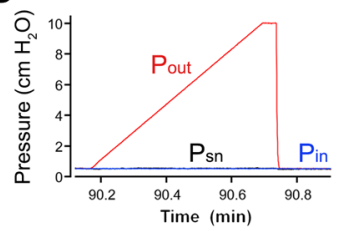

C
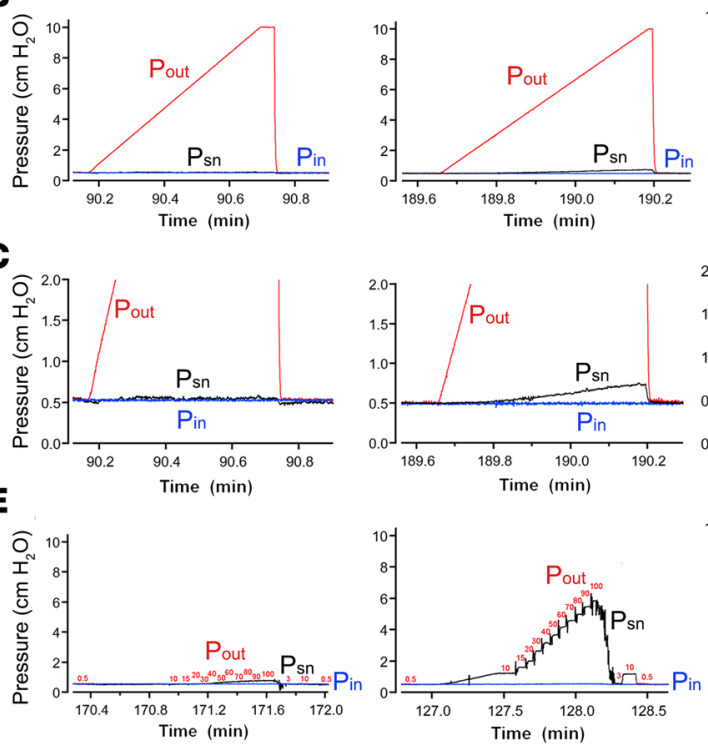

FOXC2 $^{+/ \text {CreERT2 }}$
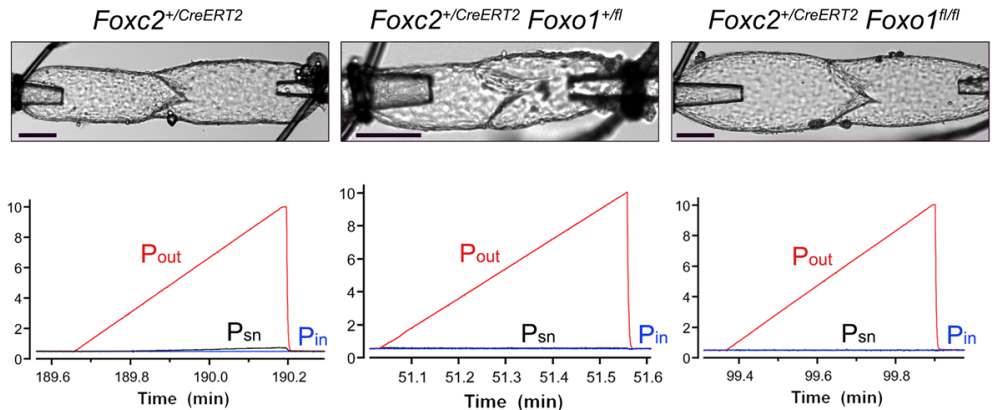

Time (min)
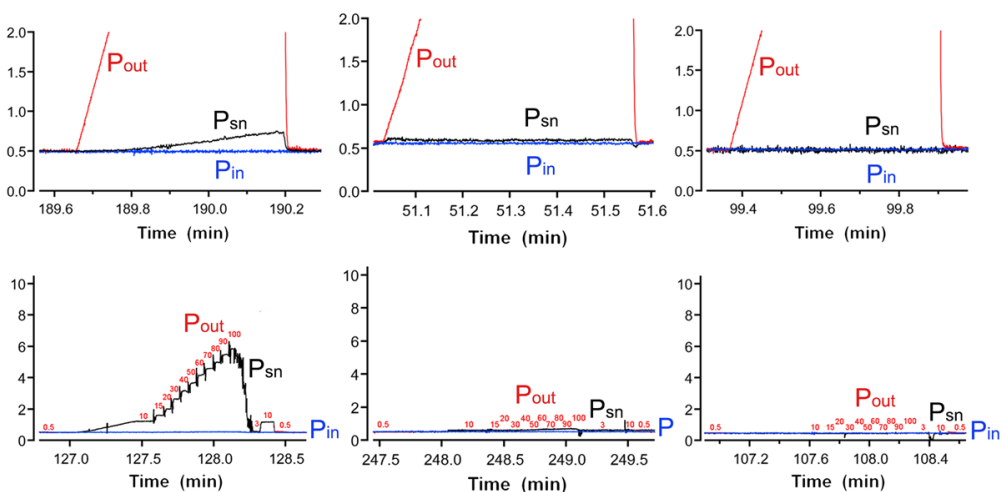

○ Control

口 FOXC2 $2^{+/ C r e E R T 2}$

$\triangle$ FOXC2 $2^{+/ C r e E R T 2}$ FOXO1 $1^{\text {th }}$

$\nabla$ FOXC2 $2^{+/ \text {CreERT2 FOXO1 }} 1^{\text {H/ffl }}$
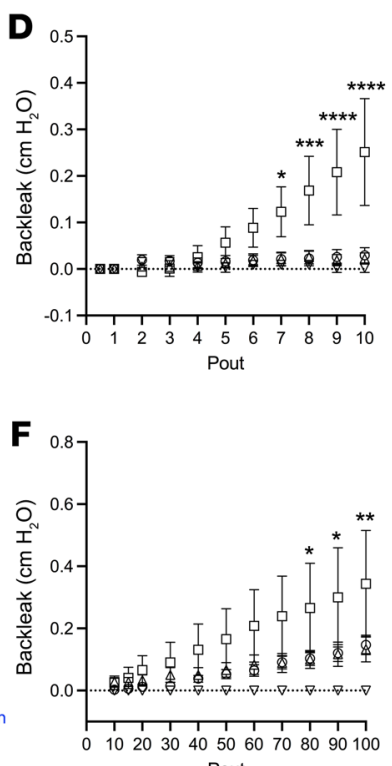

Pout

Figure 7. Loss of Foxo1 restores the function of valves in Foxc2 heterozygous mice. (A) The morphology of representative valves in the valve function test. (B) Raw traces of input pressure $\left(P_{\text {in }}\right)$, output pressure $\left(P_{\text {out }}\right)$, and the pressure right behind the valve leaflets $\left(P_{\text {sn }}\right)$ during the low pressure backleak tests $\left(0.5-10 \mathrm{cmH}_{2} \mathrm{O}\right)$ for each of the indicated genotypes. (C) Zoomed-in views of the raw traces in $\mathbf{B}$ to show changes in $P_{\text {sn }}$. (D) Summary data of the average backleak $\left(\mathrm{cmH}_{2} \mathrm{O}\right)$ for each genotype during the low pressure backleak tests. (E) Raw pressure traces during the high pressure backleak tests (10-100 $\mathrm{cmH}_{2} \mathrm{O}$ ) to show changes in $\mathrm{P}_{\text {sn }}$. The $\mathrm{P}_{\text {in }}$ and $\mathrm{P}_{\text {out }}$ traces did not change during this test because $\mathrm{P}_{\text {in }}$ was held constant and $\mathrm{P}_{\text {out }}$ was changed manually to the indicated pressures (red text). (F) Summary data of the average backleak $\left(\mathrm{cmH}_{2} \mathrm{O}\right)$ for each genotype during the high pressure backleak tests. All values are mean \pm SEM of $n=9-16$ valves from 8 animals of each genotype. Two-way ANOVA was performed with Tukey's multiple comparisons test $\left({ }^{*} P<0.05\right.$, ${ }^{* *} P$ $<0.01,{ }^{* *} P<0.001$, and $\left.{ }^{* * * *} P<0.0001\right)$.

valve-forming areas were positive for KLF4 (Figure 3, I-L). In addition to the valve transcription factors, we also checked the expression of integrin $\alpha 9$, which is critical for the assembly of the matrix core of the valve leaflets and a hallmark of a mature valve $(12,60)$. Both the mature valves and the valve-forming areas expressed high levels of integrin $\alpha 9$, which further confirmed that these valve-forming areas were undergoing normal valve morphogenesis to become mature (Figure 3, M-P). Smooth muscle cell (SMC) coverage is a feature of mature collecting lymphatic vessels (61) and mural cells are responsible for initiating the pumping of the vessels to move lymph forward in various tissue types (5). Although the lymphatic vessels in the mouse mesentery generally do not contract (62), SMCs are still recruited to these vessels with decreased SMC coverage around the valves $(63,64)$. Our SMA staining showed that similar to the valves in the control mesentery, the valve areas in the Foxo1 ${ }^{\text {LEC-KO }}$ also had diminished SMC coverage (Figure 3, Q-T). A similar phenotype of SMC coverage was observed in the Cdh5-CreER $R^{T 2}$ Foxo $1^{f / / f l}$ (Supplemental Figure 5, O-R) and in the Prox1CreERT2 Foxo1 ${ }^{\mathrm{fl} / \mathrm{fl}}$ Foxo $3^{f / f l}$ vessels (Supplemental Figure 7, G-J). Junctional proteins between the endothelial cells maintain the integrity of the vessels and some also serve as signaling molecules to regulate the expression of downstream genes. We recently published that the adherens junction protein, VE-cadherin, is not only essential for maintaining lymphatic vessel integrity, but also regulates lymphatic valve development in response to oscillatory FSS (36). Moreover, the expression of the tight junction protein Claudin 5 (CLDN5) was reported to be repressed by FOXO1 in BECs $(65,66)$, but it is unknown whether deletion of Foxo1 affects CLDN5 expression in LECs. Therefore, we performed whole-mount immunostaining of the junctional proteins VE-cadherin, PECAM1, CLDN5, and $\beta$-catenin in the mesenteries from Foxo1 ${ }^{L E C-K O}$ animals and controls. Our images showed that the expression levels and the localization of the junction proteins did not differ between the mature valves and the valve-forming areas (Figure 3, U-BB), which is consistent with our data that the Foxo1 ${ }^{\mathrm{LEC}-K O}$ animals did not exhibit lymph leakage, chylous ascites, or edema.

Loss of FOXO1 promotes lymphatic valve formation by upregulating the expression of valve-forming genes FOXC2, GATA2, KLF2, and KLF4. So far, we have shown that loss of Foxo1, but not Foxo3, from LECs induced additional valve formation. Several publications have described that deletion of Foxo1 results in increased proliferation in BECs and LECs $(42,44)$. To investigate whether proliferation is involved in our enhanced valve formation phenotype in the Foxo1 ${ }^{L E C-K O}$ animals, Ki67 (proliferation marker) and FOXC2 (valve gene) whole-mount immunostaining was performed in the mesentery following the same tamoxifen injection procedure as in Figure 2A. We quantified the number of Ki67 and FOXC2 double-positive cells in the valves and in the non-valve LECs 
from the Foxo1 ${ }^{L E C-K O}$ and control mice. Confocal imaging showed that Foxo1 ${ }^{L E C-K O}$ and control valves exhibited a similar number of proliferating cells in both the valve and non-valve LECs (Supplemental Figure 8). Therefore, postnatal deletion of Foxo1 did not significantly alter the proliferation rate of LECs and an increased proliferation rate, over the baseline rate, was not the cause for the growth of new valves.

As a transcription factor, FOXO1 can directly repress downstream gene expression. To investigate the transcriptional regulation of FOXO1 in the lymphatic valve formation, we used lentiviral shRNA against FOXO1 to efficiently knock it down in cultured human dermal lymphatic endothelial cells (hdLECs) and examined the expression levels of known valve-forming and FSS genes FOXC2, GATA2, PROX1, KLF2, and KLF4 using quantitative realtime PCR (qRT-PCR) assay. Since it is unknown whether oscillatory shear stress (OSS) regulates FOXO1 expression directly and it is also possible that OSS and FOXO1 cooperatively regulate valve-forming genes, we tested the effect of OSS and shFOXO1 in 4 groups: (a) hdLECs cultured with scramble shRNA and no OSS (static control); (b) hdLECs treated with shFOXO1 but no OSS; (c) hdLECs exposed to OSS with scramble shRNA (OSS control); and (d) hdLECs treated with shFOXO1 under OSS. Our results showed that while FOXO1 mRNA levels did not change in response to OSS, the expression levels of FOXC2, GATA2, KLF2, and KLF4 were all significantly $(P<0.05)$ increased under OSS, which is consistent with previous reports (Figure $4, \mathrm{~A}-\mathrm{F}$, closed circles) $(15,36,51,58,59)$. More importantly, the expression levels of FOXC2, GATA2, KLF2, and KLF4 were significantly $(P<$ 0.05) upregulated in the cells treated with shFOXO1 under static conditions, which supports our hypothesis that FOXO1 represses the valve-gene program (Figure 4, A-F, open circles). Moreover, shFOXO1 combined with OSS further increased the expression levels of the valve-forming genes, indicating that FOXO1 is not the only effector by which OSS regulates valve-forming genes (Figure 4, A-F). To further support the qRT-PCR results, Western blot of FOXC2, GATA2, KLF4, and FOXO1 was performed to show the protein expression levels of these genes while $\beta$-ACTIN was used as the loading control. Consistent with the mRNA levels, FOXO1 was successfully knocked down and FOXC2, GATA2, and KLF4 protein were upregulated in response to shFOXO1 and OSS (Figure $4 \mathrm{G}$ ).

Given the 3 observations so far that AKT attenuates nuclear FOXO1 activity by phosphorylation in many cell types; AKT is activated upon OSS, which is a driver for valve formation; and loss of FOXO1 activity results in new valve formation, the role of the OSS/ AKT/FOXO1 signaling axis was investigated in hdLECs. Immunostaining of the cells showed that the localization of FOXO1 changed from nucleus to cytoplasm in response to OSS (Figure $4, \mathrm{H}-\mathrm{K})$, which is consistent with the Western blot results that the levels of phosphorylated AKT and FOXO1 were both increased upon OSS (Figure 4L). To further analyze the role of AKT in FOXO1 phosphorylation, the AKT activator SC-79 was used to activate AKT and a Western blot of phosphorylated FOXO1 was performed. As expected, the levels of phosphorylated AKT and FOXO1 were both increased in the presence of SC-79 (Figure 4M). These results indicated that nuclear FOXO1 activity was reduced by AKT in response to OSS.
To identify the direct target of FOXO1 during valve formation, FOXO1 DNA binding sites T[G/A]TTTAC and T[G/A]TTTTG were found in the promoter area $(0.3 \mathrm{~kb}$ upstream of the start codon) of the FOXC2 gene (Figure $4 \mathrm{~N}$ ). Chromatin immunoprecipitation (ChIP) assay was performed to confirm the binding of FOXO1 in this particular area. Two other nonbinding promoter areas $(-1.4 \mathrm{~kb}$ and $-2.7 \mathrm{~kb})$ were used as controls since no other FOXO1 binding sites were found within the $4 \mathrm{~kb}$ upstream area of the FOXC2 start codon. By comparing the DNA amount bound by IgG and FOXO1, ChIP data showed that indeed FOXO1 bound on the $-0.3 \mathrm{~kb}$ area in the FOXC2 promoter, but not the $-1.4 \mathrm{~kb}$ and $-2.7 \mathrm{~kb}$ areas, which supports the conclusion that FOXO1 represses FOXC2 expression by direct regulation (Figure 4, O-Q).

Ablation of Foxo1 rescues the valve loss in a mouse model of lymphedema. Although lymphatic valves are required for lymph to move forward, and valve loss or dysfunction is strongly associated with lymphedema, it is unknown whether valve loss or dysfunction is irreversible. Here, we took advantage of the $\mathrm{Foxc2}^{+-}$mice, which are a model of the human disease lymphedema distichiasis (17, 19). We obtained a knock in-knock out Foxc2 heterozygous mouse, which replaces the open reading frame of Foxc2 with the cDNA for $\mathrm{CreER}^{\mathrm{T} 2}$. Therefore, this mouse strain is heterozygous for Foxc2 and expresses a TM-inducible Cre in all postnatal LECs (67). Wholemount immunostaining indicated that P1, P3, and P5 TM injections lead to approximately $100 \%$ efficient deletion of FOXO1 in all LECs (Supplemental Figure 1, I-N). We bred the Foxc2 ${ }^{+/ \text {CreERT2 }_{2}}$ mice with the Foxo1 $1^{f / f l}$ mice to obtain Foxc2 $2^{+/ C r E R T 2}$ Foxol $^{+/ f l}$ and Foxc2 $2^{+/ C r e E R T 2}$ Foxo1 ${ }^{\text {t/fll }}$ mice. We also bred the LEC reporter line Prox1-GFP for visualizing the lymphatic vasculature. This mouse model allowed us to delete 1 or 2 alleles of Foxo1 from all LECs on a Foxc2 ${ }^{+/-}$background. Mesenteric lymphatic vessels in the Foxc2 ${ }^{+/ C r E R T 2} \mathrm{Foxo1}^{+/ f l}$,

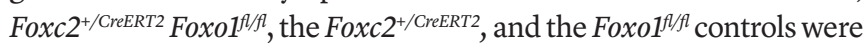
analyzed at P21 (Figure 5A). After we counted the valve number and branch points in all 4 genotypes, our data revealed the following results: (a) Foxc2 ${ }^{+/ \text {CreERT2 }}$ animals lack $50 \%$ valves in the mesentery compared with the controls and the number of branches was significantly $(P<0.0001)$ reduced; (b) complete deletion of Foxo1 in

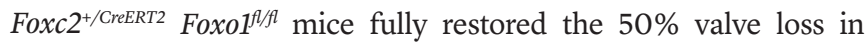
Foxc2 $2^{+/ \text {CreERT2 }}$ to control levels by P21; (c) complete deletion of Foxo1

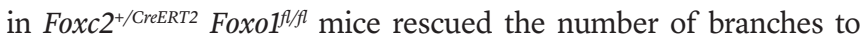
control levels; and (d) one allele deletion of Foxo1 did not rescue the valve and branch number in the Foxc2 ${ }^{+/ \text {CreERT2 }}$ mice (Figure 5, B-E). To further confirm that the morphology of valves and the expression of valve-specific genes were restored in Foxc2 ${ }^{+/ \text {CreERT2 }} \mathrm{Foxo}^{\mathrm{fl} / \mathrm{l} l}$ animals, we performed whole-mount immunostaining of PROX1, FOXC2, VE-cadherin, and integrin $\alpha 9$ in all 4 genotypes. Figure 6, A-H showed that although some of the valves in the Fox $2^{+/ \text {CreERT2 }}$ and Foxc2 $2^{+/ \text {CreERT2 }}$ Foxo1 $^{+/ f l}$ animals expressed PROX1 and FOXC2, they lack the valve leaflets indicated by integrin $\alpha 9$ expression compared with controls. The percentage of integrin $\alpha 9$-positive valves was significantly $(P<$ 0.05) lower in both the Foxc2 $2^{+/ \text {CreERT2 }}$ and Foxc2 $2^{+/ \text {CreERT2 }}$ Foxo1 $^{+/ f l}$ mice than in the Foxo $1^{f / / f l}$ controls and Foxc2 ${ }^{+/ C r e E R T 2}$ Foxo1 $1^{f / / l}$ animals (Figure 6I). Intriguingly, the loss of valve leaflets and the expression of integrin $\alpha 9$ were restored by complete ablation of Foxo1 in the Foxc2 ${ }^{+/ C r E R T 2}$ animals (Figure 6, D and H). Strikingly, these data demonstrate that valve loss in a lymphedema mouse model can be rescued after regression or impaired development has occurred. 
Ablation of Foxo1 rescues valve function in a mouse model of lymphedema. It has been reported that the function of the valves in the Foxc2 $2^{+/ C r e E R T 2}$ animals is defective (68). To investigate whether Foxo1 deletion not only rescues the morphology and the number of the valves in the Foxc2 $2^{+/ C r e E R T 2}$ animals, but also the function of the valves in those animals, a previously reported valve function test was performed in the Foxc2 $2^{+/ C r E R T 2}, \mathrm{Foxc2}^{+/ \mathrm{CreERT}_{2}} \mathrm{FoxOl}^{+/ f l}$, Foxc2 ${ }^{+/ C r e E R T 2}$ Foxol ${ }^{l / f l}$, and control mice (Foxo $1^{f / f l}$ and WTs; refs. 11, 68). Single lymphatic vessel segments containing 1 valve were surgically excised from the mouse mesentery and then tied onto 2 glass pipettes that enabled fine pressure control over each end of the vessel. To test the ability of each valve to seal against pressure, the downstream (i.e., output) pressure was gradually raised from 0.5 $\mathrm{cmH}_{2} \mathrm{O}$ to $10 \mathrm{cmH}_{2} \mathrm{O}$, while keeping the upstream (i.e., input) pressure constant. A smaller pipette inserted in the vessel lumen right behind the valve leaflets measured the pressure leaking backwards through the valve (not shown). Thus, this test measured the amount of backleak through the valve leaflets. Single valves obtained from WT and Foxo1 ${ }^{l / f l}$ controls, Foxc2 ${ }^{+/ C r e E R T 2}$, Foxc2 $2^{+/ \text {CreERT2 }}$ Foxo1 $^{+/ f l}$, and Foxc2 $^{+/ C r E R T 2}$ Foxo $^{f / / f l}$ mice appeared morphologically indistinguishable (Figure 7A) due to the inability to choose morphologically defective valves. When downstream pressure was elevated to 10 $\mathrm{cmH}_{2} \mathrm{O}$, there was noticeable pressure backleak in the Foxc2 $2^{+/ \text {CreERT2 }}$ valves (Figure 7, B and C, black traces). In contrast, there was no pressure backleak across the Foxc2 ${ }^{+/ C r e E R T 2} \mathrm{FoxO}^{+/ f l}$ or Foxc2 $2^{+/ C r e E R T 2}$ Foxo1 $1^{f / f l}$ valve leaflets when pressure was elevated to a physiological pressure of $10 \mathrm{cmH}_{2} \mathrm{O}$ (Figure 7, B and C). Therefore, loss of either 1 or 2 alleles of Foxo1 led to a significant rescue in valve function in the Foxc2 heterozygous valves over low physiological pressures (Figure 7D). Because subtle defects in valve sealing are magnified at higher pressures, we tested the same valves to $100 \mathrm{cmH}_{2} \mathrm{O}$ (Figure 7, E and F). Indeed, the amount of backleak in the Foxc2 ${ }^{+/ \text {CreERT2 }}$ valves peaked at approximately $6 \mathrm{cmH}_{2} \mathrm{O}$ when output pressure was raised to $100 \mathrm{cmH}_{2} \mathrm{O}$. At pressures that high, some control valves eventually failed and began to leak (data not shown). While the Foxc2 $2^{+/ C r e E R T 2}$ Foxo1 ${ }^{+/ f l}$ valves were not significantly different from control valves, remarkably, none of the Foxc2 $2^{+/ \text {CreERT2 }}$ Foxo1 $^{\text {fl/fl }}$ valves exhibited any backleak even when facing a pressure of $100 \mathrm{cmH}_{2} \mathrm{O}$ (Figure 7F). Thus, these experiments show that loss of Foxo1 enhances valve function in a mouse model of lymphedema distichiasis.

\section{Discussion}

Previously, we discovered that VE-cadherin plays an essential role in lymphatic valve development by initiating mechanotransduction signaling through the $\beta$-catenin and AKT pathways in response to OSS (36). In that study, we unexpectedly found that a chemical activator of AKT stimulated de novo valve growth in control mice. FOXO1 is a transcription factor regulated by AKT, although its role in the lymphatic vasculature remained unclear. Here, we identified Foxo1 as a key negative regulator of lymphatic valve formation by repressing valve-specific genes and that LEC-specific or vascular-specific deletion of Foxo1 caused significantly $(P<0.01)$ more lymphatic valves to form throughout postnatal development. Furthermore, loss of Foxo1 in a mouse model of the human disease lymphedema-distichiasis fully restored the number of valves and valve function to normal control levels, indicating that this transcription factor holds clinically relevant value.
FOXO1 inhibits lymphatic valve formation by repressing valve gene expression. Many studies have shown that lymph flow is oscillatory at the valve areas and it is this OSS from lymph flow that provides constant cellular survival signals that stimulate the formation and continuous maintenance of the valve leaflets by upregulating valve genes (e.g., FOXC2, GATA2, CTNNB1, ITGA9, etc.) in LECs to stimulate the formation and lifelong maintenance of valves $(15,51,58,59)$. Loss of lymph flow in vivo prevents the onset of valve formation (58). Here, using both in vivo and in vitro approaches, we revealed that FOXO1 repressed the valve-forming gene program because: (a) the nuclear localization of FOXO1 (i.e., FOXO1 activity) was absent in embryonic valve-forming cells; (b) the localization of FOXO1 changed from nucleus to cytoplasm in response to OSS; and (c) knockdown of FOXO1 in cultured LECs upregulated the expression of many valve- and shear-responsive genes (i.e., FOXC2, GATA2, KLF4, and KLF2). These data indicate that by removing FOXO1, the expression of valve-forming genes was enhanced, leading to new valve growth. By rigorously counting all the valves in the mesentery at multiple developmental stages, we demonstrated that there is indeed an increase in fully mature valves. By performing ChIP assay, we have identified FOXC2 as a direct target of FOXO1. FOXO1 binds directly to the promoter area of FOXC2 and represses its expression. Thus, it will be informative to identify more valve genes as the direct or indirect downstream targets of FOXO1 by ChIP in the future. Our data suggest that FOXO1 might not be the only repressor of lymphatic valve formation. The reasons include that loss of Foxo1 did not cause uncontrolled growth of new valves and although the number of valves per millimeter was increased, the newly formed valves were still spaced apart from each other. When Foxo1 was deleted postnatally after many valves had already formed, the leaflets of the preexisting valves did not become longer. Therefore, more research is required to identify other genes that regulate the spacing of the valves and the length of the valve leaflets.

Rescue of valve formation and function in a lymphedema mouse model. Although defective valves are strongly associated with lymphedema, there are no existing therapeutic strategies to grow new valves. Currently, it is not even known whether valve dysfunction/regression is reversible. In a previous study, overexpression of the main valve-specific gene, Foxc2, failed to induce additional valve formation (59), which suggests that multiple genes may need to be targeted simultaneously to grow valves. A potential solution to this problem is to identify a transcriptional repressor of the valve-forming gene program, which would be a highly desired therapeutic target to ameliorate valve defects in patients with lymphedema. Previous work on lymphatic valve development has only discovered positive regulators of valve formation that cause valve regression when deleted $(12,15,23,51,59,63,69,70)$. In this study, we successfully grew more lymphatic valves by targeting a single gene and rescued the valve phenotype in a mouse model of lymphedema.

The valve-forming gene Foxc2 is one of the key regulators for both the formation and maintenance of lymphatic valves $(10,51)$, and Foxc2 heterozygous $\left(F o x c 2^{+-}\right)$mice are a model of the human disease lymphedema distichiasis $(10,17) . F_{0 x c 2^{+/-}}$mice survive into 
adulthood, but their lymphatic vessels develop 50\% fewer valves than WT vessels, and the remaining valves have shorter valve leaflets $(19,45,51)$. The short length prohibits tight sealing of the valves and leads to retrograde flow $(51,68)$. We showed that the complete loss of Foxol on the Foxc2 ${ }^{+/-}$heterozygous background restored the morphology of the defective valves to healthy control levels, most likely by increasing the levels of valve-forming genes enough to restore valve growth. While Foxo1 was deleted in every lymphatic endothelial cell, it appeared that this approach only affected the defective valves. Ablation of Foxol from Foxc2 ${ }^{+-}$mice also rescued the defects in branching to control levels, and postnatal deletion of Foxo1 did not induce lymphangiogenesis in the control mesenteries. In conclusion, Foxo1 deletion leads to the formation of new additional valves that are morphologically and molecularly normal, and can rescue loss of valves in lymphedema mouse models. In addition to the morphological rescue, Foxo1-deficient valves exhibit enhanced valve function because they can rescue valve backleak found in Foxc2 heterozygous mice with deletion of only a single allele of Foxo1. Thus, Foxo1 may represent a valuable clinical target for the treatment of lymphedema because it does not cause uncontrolled growth of the healthy vessels or valves, but selectively restores defective lymphatic valves.

Long-term deletion of Foxo1. Although Prox1 is expressed exclusively in the LECs within the vasculature, Prox1 is also expressed in the brain, liver, eye, skeletal muscle, and pancreas along with Foxo1 (52-55). Thus, the Prox1CreER ${ }^{T 2}$ line not only deletes Foxo1 from the LECs but also from the PROX1/FOXO1 double-positive cells in the brain, liver, eye, skeletal muscle, and pancreas, which might cause additional phenotypes. Although a systematic study of the effect for long-term deletion of Foxo1 using Prox1CreER $R^{T 2}$ was not conducted, 5 pairs of the Foxo1 ${ }^{L E C-K O}$ mice and their littermate controls were observed for up to 12 months. There was no obvious difference between the controls and the knockouts in terms of weight at 12 months and their life span. No eye defects including blindness, cataracts, or exophthalmos were observed in these animals. Nevertheless, to strengthen the clinical value of targeting Foxo1, a systematic study of how long-term deletion of Foxo1 affects multiple organs is needed in the future.

Role of FOXO1 in endothelial cell growth. As an important component in the metabolic pathway of growth factors, Foxo1 has been shown to regulate cell proliferation in many cell types $(71,72)$. Embryonic deletion of Foxo1 results in overgrowth of the retinal blood vasculature caused by enhanced proliferation while overexpression of Foxo1 restricts endothelial growth and vascular expansion (42). Increased proliferation was also reported in the dermal lymphatic vessels in the mouse tail after Foxo1 deletion (44). These results are consistent with our finding that the diameter of the mesenteric lymphatic vessels was increased during embryonic deletion of Foxo1 in the LECs. To investigate the role of proliferation in new valve growth, we measured the proliferation status of the valve cells and non-valve lymphangion cells using Ki67 staining in the postnatal pups. In contrast to embryonic deletion, postnatal deletion of Foxo1 did not affect the diameter or the branch number of the mesenteric and axillary lymphatic vessels. No excessive growth of the lymphatic vasculature was observed in either the mesentery or the skin. Moreover, the number of Ki67-positive cells was similar in the valve cells and in the non-valve cells. These results support that FOXO1 represses valve formation mainly through its transcriptional regulation of the valve-forming gene program. Interestingly, we did observe a different morphology of the lymphatic vessels in the postnatal ear after we deleted Foxo1. The lymphatic vessels in the ear after 21 days of deletion were enlarged and many of them lacked smooth muscle cell coverage, whereas the vessels in the mesentery remained the same diameter and retained normal smooth muscle cell coverage. The number of Ki67-positive cells was also increased in the ear lymphatics. The difference in the phenotype of the vessels in the mesentery and in the ear could be caused by the time point for Foxo1 deletion. Postnatal deletion of Foxo1 was introduced right after birth when the mesenteric lymphatic vessels had already formed. However, this same time point is when the ear lymphatic vessels are just at the beginning of formation. Furthermore, the dilation of the ear lymphatics was consistent with the deletion of Foxo1 in the embryos when the mesenteric vessels were still forming. Therefore, Foxo1-mediated signaling pathways could have different effects at different developmental stages.

In summary, our study reported what we believe are novel findings of Foxo1 deletion in the lymphatic endothelial cells. By deleting this single gene, we successfully activated the lymphatic valve formation process in otherwise normal vessels. More importantly, inactivation of Foxo1 in a lymphedema mouse model completely rescued the defective morphology, number of valves, and valve function. The findings reveal that as a key downstream effector of shear stress, Foxo1 activity is crucial for controlling the expression of many valve-forming genes, and thus represents a potential therapeutic target for treating primary lymphedema patients.

\section{Methods}

Mice. The Prox1CreER ${ }^{T 2}$, Cdh5(PAC)-CreERT2, Foxo1 ${ }^{f l}$, Foxo3 $^{f l}$, Prox1GFP, and Foxc2 ${ }^{+/ C r e E R T 2}$ strains were reported previously (47-49, 56, 67, 73). The Prox1CreER ${ }^{T 2}$, Cdh5(PAC)-CreERT2, Prox1-GFP, and Foxc2 $2^{+/ C r E R T 2}$ strains were obtained through material transfer agreement (MTA) with the investigators who developed the mouse models. The Foxo1 ${ }^{f l}$ and $\mathrm{Foxo}^{f l}$ strains were obtained from the Jackson Laboratory. The Prox1CreER ${ }^{T 2}$, Cdh5(PAC)-CreERT2, Foxo ${ }^{f l}$, Foxo $3^{f l}$, Prox1-GFP were maintained on a mixed genetic background (NMRI $\times \mathrm{C} 57 \mathrm{BL} / 6 \mathrm{~J} \times \mathrm{FVB})$ and both sexes were used. The Foxc2 $2^{+/ C r e E R T 2}$ and the Foxc2 ${ }^{+/ \text {CreERT2 }_{2}} \mathrm{Foxo1}^{l l}$ mice were maintained on a pure C57BL/6J background and both sexes were used. Embryonic deletion was induced by a single intraperitoneal injection of $5 \mathrm{mg}$ tamoxifen into pregnant dams at E14.5. Pups were injected subcutaneously with 100 $\mu \mathrm{g}$ tamoxifen in $5 \mu \mathrm{L}$ sunflower oil on postnatal days P1 and P3 or P1, P3, and P5. Adult mice were injected intraperitoneally with $1 \mathrm{mg}$ tamoxifen in week 7 for 5 consecutive days.

Ex vivo analysis of lymphatic vasculature and lymphatic valve quantification. Embryos and postnatal pups expressing the Prox1GFP reporter strain were euthanized at the indicated developmental stages. GFP ${ }^{\text {hi }}$ lymphatic valves in both the large collecting lymphatic vessels and those thinner branched precollecting vessels located closer to the intestinal wall (from the lymph node to the intestinal wall) (46) were counted in situ (without being excised out of the body) under a Zeiss V16 microscope in each section of the mesentery using a hand tally counter beginning at the duodenum and terminating at the cecum. The number of valves from each section of the mesentery 
were added together to obtain the total number of valves. The examples of mature and immature valves were indicated in Figure 2, D, E, H, I, L, and M. Four control and 4 knockout animals were used from each mesentery. Additionally, 4 more analyses-valves per millimeter, number of branches, intervalve distance, and lymphatic vessel diameter-were performed. The complete procedures are included in the Supplemental Methods.

Quantification of cell proliferation in the lymphatic vessels and smooth muscle cell coverage. The complete procedures are included in the Supplemental Methods.

Whole-mount immunostaining procedure. The protocol of wholemount immunostaining was previously reported (36). The complete procedure is included in the Supplemental Methods.

Antibodies. The full list of antibodies is included in the Supplemental Methods.

Cell culture, shRNA, and OSS. Primary hdLECs (PromoCell, C-12216) were maintained in EBM-2 MV media (PromoCell, C-22121) on fibronectin-coated 6-well plates. Cells at passage 6 or lower were used for shRNA knockdown and the OSS assay. To knock down FOXO1, cells were infected with lentiviral particles expressing an shRNA targeting FOXO1 or a control scramble construct expressing GFP for 48 hours. The OSS assay was performed according to our previously published protocol $(36,59)$. When the cells reached confluency in 6-well plates, they were exposed to OSS using a test tube rocker (Thermolyne Speci-Mix aliquot mixer model M71015, Barnstead International) in a humidified cell culture incubator with $5 \% \mathrm{CO}_{2}$ for 48 hours. The lentiviral shFOXO1 was purchased from VectorBuilder under shFOXO1\#2, and the sequence of shFOXO1 is CAGGACAATAAGTCGAGTTAT.

For SC-79 treatment, hdLECs were cultured to reach over 95\% confluency. The medium was switched from EBM-2 MV containing 5\% serum to $0.5 \%$ serum medium for starvation. After 6 hours of starvation, $8 \mu \mathrm{g} / \mathrm{mL}$ SC-79 (Millipore) was added into the medium for 30 minutes before total protein was harvested. DMSO treatment was used as control.

RNA isolation, qRT-PCR, Western blot, and ChIP assay. The complete procedures are included in the Supplemental Methods.

Vessel isolation and cannulation. The procedure of vessel isolation and cannulation was previously reported (74). The complete protocol is included in the Supplemental Methods.
Valve function tests. The procedure of valve function tests was previously reported (11). The complete protocol is included in the Supplemental Methods.

Statistics. All data are represented as mean \pm SEM. For data sets containing 2 groups, an unpaired 2-sided Student's $t$ test was used to determine significant differences $(P<0.05)$. For data sets with 1 independent variable, 1-way ANOVA with Tukey's multiple comparisons test was performed to test for significant differences, where $P$ less than 0.05 was considered significant. For data sets with 2 independent variables, 2-way ANOVA with Tukey's multiple comparisons tests were performed to determine significant differences $(P<0.05)$. Graphpad Prism software (version 6) was used for all statistical analyses and to plot quantitative data.

Study approval. All animal experiments were performed in accordance with the University of South Florida or University of Missouri guidelines and were approved by the respective IACUC committees. All procedures complied with the standards stated in the Guide for the Care and Use of Laboratory Animals (National Academies Press, 2011).

\section{Author contributions}

YY performed experiments, analyzed data, and wrote the manuscript. JPS developed analytical tools and wrote the manuscript. LAK, HH, JACG, and MJD performed experiments and edited the manuscript. All authors contributed to and approved the final version of the manuscript.

\section{Acknowledgments}

We thank Taija Makinen for sharing the Prox1CreER ${ }^{T 2}$ mice; Young Kwon Hong for sharing the Prox1-GFP mice; R. Sathish Srinivasan for sharing the Foxc2 $2^{+/ C r E R T 2}$ mice; and Ralf Adams for sharing the Cdh5(PAC)-CreERT2 mice. We thank Hana Totary-Jain for sharing the quantitative RT-PCR machine. This work was supported by National Heart, Lung, and Blood Institute (NHLBI) grants R01 HL145397 (to YY) and R01 HL122578 (to MJD), and NIH grant R01 HL142905 (to JPS).

Address correspondence to: Ying Yang, 12901 Bruce B. Downs Boulevard, MDC08, Tampa, Florida 33612, USA. Phone: 813.974.8078; Email: yingyang@usf.edu.
1. Yang Y, Oliver G. Development of the mammalian lymphatic vasculature. JClin Invest. 2014;124(3):888-897.

2. Koltowska K, et al. Getting out and about: the emergence and morphogenesis of the vertebrate lymphatic vasculature. Development. 2013;140(9):1857-1870.

3. Tammela T, Alitalo K. Lymphangiogenesis: molecular mechanisms and future promise. Cell. 2010;140(4):460-476.

4. Breslin JW, et al. Lymphatic vessel network structure and physiology. Compr Physiol. 2018;9(1):207-299.

5. Scallan JP, Davis MJ. Genetic removal of basal nitric oxide enhances contractile activity in isolated murine collecting lymphatic vessels. J Physiol. 2013;591(pt 8):2139-2156.

6. Scallan JP, et al. Constriction of isolated collecting lymphatic vessels in response to acute increases in downstream pressure. J Physiol. 2013;591(pt 2):443-459.

7. Szuba A, Rockson SG. Lymphedema: classification, diagnosis and therapy. Vasc Med. 1998;3(2):145-156.

8. Neligan PC, et al. MR lymphangiography in the treatment of lymphedema. J Surg Oncol. 2017;115(1):18-22

9. Brorson H. Liposuction in arm lymphedema treatment. Scand J Surg. 2003;92(4):287-295.

10. Petrova TV, et al. Defective valves and abnormal mural cell recruitment underlie lymphatic vascular failure in lymphedema distichiasis. Nat Med. 2004;10(9):974-981.

11. Lapinski PE, et al. RASA1 regulates the function of lymphatic vessel valves in mice. J Clin Invest. 2017;127(7):2569-2585.

12. Bazigou $\mathrm{E}$, et al. Integrin-alpha9 is required for fibronectin matrix assembly during lymphatic valve morphogenesis. Dev Cell. 2009;17(2):175-186.

13. Ma GC, et al. A recurrent ITGA9 missense mutation in human fetuses with severe chylothorax: possible correlation with poor response to fetal therapy. Prenat Diagn. 2008;28(11):1057-1063.

14. Ostergaard P, et al. Mutations in GATA2 cause primary lymphedema associated with a predisposition to acute myeloid leukemia (Emberger syndrome). Nat Genet. 2011;43(10):929-931.

15. Kazenwadel J, et al. GATA2 is required for lymphatic vessel valve development and maintenance. JClin Invest. 2015;125(8):2979-2994.

16. Martin-Almedina S, et al. EPHB4 kinase-inactivating mutations cause autosomal dominant lymphatic-related hydrops fetalis. J Clin Invest. 2016;126(8):3080-3088.

17. Fang J, et al. Mutations in FOXC2 (MFH-1), a forkhead family transcription factor, are responsible for the hereditary lymphedema-distichiasis syndrome. Am JHum Genet. 2000;67(6):1382-1388.

18. Brice G, et al. Analysis of the phenotypic abnormalities in lymphoedema-distichiasis syndrome in 74 patients with FOXC2 mutations or linkage 
to 16q24. JMed Gentet. 2002;39(7):478-483.

19. Kriederman BM, et al. FOXC2 haploinsufficient mice are a model for human autosomal dominan lymphedema-distichiasis syndrome. Hum Mol Genet. 2003;12(10):1179-1185.

20. Dagenais SL, et al. Foxc2 is expressed in developing lymphatic vessels and other tissues associated with lymphedema-distichiasis syndrome. Gene Expr Patterns. 2004;4(6):611-619.

21. Burrows PE, et al. Lymphatic abnormalities are associated with RASA1 gene mutations in mouse and man. Proc Natl Acad Sci U S A. 2013;110(21):8621-8626.

22. Ferrell RE, et al. GJC2 missense mutations cause human lymphedema. Am J Hum Genet. 2010;86(6):943-948.

23. Kanady JD, et al. Connexin 37 and Connexin 43 deficiencies in mice disrupt lymphatic valve development and result in lymphatic disorders including lymphedema and chylothorax. Dev Biol. 2011;354(2):253-266.

24. Brice G, et al. A novel mutation in GJA1 causing oculodentodigital syndrome and primary lymphoedema in a three generation family. Clin Genet. 2013;84(4):378-381.

25. Lukacs V, et al. Impaired PIEZO1 function in patients with a novel autosomal recessive congenital lymphatic dysplasia. Nat Commun. 2015;6:8329.

26. Fotiou E, et al. Novel mutations in PIEZO1 cause an autosomal recessive generalized lymphatic dysplasia with non-immune hydrops fetalis. Nat Commun. 2015;6:8085.

27. Rasmussen JC, et al. Lymphatic imaging in humans with near-infrared fluorescence. Curr Opin Biotechnol. 2009;20(1):74-82.

28. Stanton $\mathrm{AW}$, et al. A quantitative lymphoscintigraphic evaluation of lymphatic function in the swollen hands of women with lymphoedema following breast cancer treatment. Clin Sci (Lond). 2006;110(5):553-561.

29. Miaskowski C, et al. Lymphatic and angiogenic candidate genes predict the development of secondary lymphedema following breast cancer surgery. PLoS One. 2013;8(4):e60164.

30. Avraham T, et al. Th2 differentiation is necessary for soft tissue fibrosis and lymphatic dysfunction resulting from lymphedema. FASEB $J$. 2013;27(3):1114-1126.

31. Zampell JC, et al. Temporal and spatial patterns of endogenous danger signal expression after wound healing and in response to lymphedema. $\mathrm{Am} J$ Physiol Cell Physiol. 2011;300(5):C1107-C1121.

32. Zampell JC, et al. Toll-like receptor deficiency worsens inflammation and lymphedema after lymphatic injury. Am J Physiol Cell Physiol. 2012;302(4):C709-C719.

33. Lin S, et al. Prospective transcriptomic pathway analysis of human lymphatic vascular insufficiency: identification and validation of a circulating biomarker panel. PLoS One. 2012;7(12):e52021.

34. Zampell JC, et al. Regulation of adipogenesis by lymphatic fluid stasis: part I. Adipogenesis, fibrosis, and inflammation. Plast Reconstr Surg. 2012;129(4):825-834.

35. Aschen S, et al. Regulation of adipogenesis by lymphatic fluid stasis: part II. Expression of adipose differentiation genes. Plast Reconstr Surg. 2012;129(4):838-847.
36. Yang Y, et al. VE-cadherin is required for lymphatic valve formation and maintenance. Cell Rep. 2019;28(9):2397-2412.

37. Brunet A, et al. Akt promotes cell survival by phosphorylating and inhibiting a Forkhead transcription factor. Cell. 1999;96(6):857-868.

38. Hay N. Interplay between FOXO, TOR, and Akt. Biochim Biophys Acta. 2011;1813(11):1965-1970.

39. Greer EL, Brunet A. FOXO transcription factors at the interface between longevity and tumor suppression. Oncogene. 2005;24(50):7410-7425.

40. Furuyama T, et al. Abnormal angiogenesis in Foxo1 (Fkhr)-deficient mice. J Biol Chem. 2004;279(33):34741-34749.

41. Hosaka T, et al. Disruption of forkhead transcription factor (FOXO) family members in mice reveals their functional diversification. Proc Natl Acad Sci U S A. 2004;101(9):2975-2980.

42. Wilhelm K, et al. FOXO1 couples metabolic activity and growth state in the vascular endothelium. Nature. 2016;529(7585):216-220.

43. Potente $\mathrm{M}$, et al. Involvement of Foxo transcription factors in angiogenesis and postnatal neovascularization. JClin Invest. 2005;115(9):2382-2392.

44. Niimi K, et al. FOXO1 regulates developmental lymphangiogenesis by upregulating CXCR4 in the mouse-tail dermis. Development. 2020;147(2):dev181545.

45. Sabine A, et al. Mechanotransduction, PROX1, and FOXC2 cooperate to control connexin 37 and calcineurin during lymphatic-valve formation. Dev Cell. 2012;22(2):430-445.

46. Sabine A, et al. Characterization of mouse mesenteric lymphatic valve structure and function. Methods Mol Biol. 2018;1846:97-129.

47. Bazigou E, et al. Genes regulating lymphangiogenesis control venous valve formation and maintenance in mice. JClin Invest. 2011;121(8):2984-2992.

48. Paik JH, et al. FoxOs are lineage-restricted redundant tumor suppressors and regulate endothelial cell homeostasis. Cell. 2007;128(2):309-323.

49. Choi I, et al. Visualization of lymphatic vessels by Prox1-promoter directed GFP reporter in a bacterial artificial chromosome-based transgenic mouse. Blood. 2011;117(1):362-365.

50. Norrmen C, et al. FOXC2 controls formation and maturation of lymphatic collecting vessels through cooperation with NFATc1. J Cell Biol. 2009;185(3):439-457.

51. Sabine A, et al. FOXC2 and fluid shear stress stabilize postnatal lymphatic vasculature. J Clin Invest. 2015;125(10):3861-3877.

52. Zhang W, et al. FoxO1 regulates multiple metabolic pathways in the liver: effects on gluconeogenic, glycolytic, and lipogenic gene expression. J Biol Chem. 2006;281(15):10105-10117.

53. Hoekman MF, et al. Spatial and temporal expression of FoxO transcription factors in the developing and adult murine brain. Gene Expr Patterns. 2006;6(2):134-140.

54. Kitamura T, et al. The forkhead transcription factor Foxo1 links insulin signaling to Pdx1 regulation of pancreatic beta cell growth. J Clin Invest. 2002;110(12):1839-1847.

55. Sandri M, et al. Foxo transcription factors induce the atrophy-related ubiquitin ligase atrogin-1 and cause skeletal muscle atrophy. Cell. 2004;117(3):399-412.
56. Wang Y, et al. Ephrin-B2 controls VEGF-induced angiogenesis and lymphangiogenesis. Nature. 2010;465(7297):483-486.

57. Srinivasan RS, Oliver G. Prox1 dosage controls the number of lymphatic endothelial cell progenitors and the formation of the lymphovenous valves. Genes Dev. 2011;25(20):2187-2197.

58. Sweet DT, et al. Lymph flow regulates collecting lymphatic vessel maturation in vivo. JClin Invest 2015;125(8):2995-3007.

59. Cha B, et al. Mechanotransduction activates canonical Wnt $/ \beta$-catenin signaling to promote lymphatic vascular patterning and the development of lymphatic and lymphovenous valves. Genes Dev. 2016;30(12):1454-1469.

60. Danussi C, et al. The EMILIN1/alpha9beta1 integrin interaction is crucial in lymphatic valve formation and maintenance. Mol Cell Biol. 2013;33(22):4381-4394.

61. Oliver G. Lymphatic vasculature development. Nat Rev Immunol. 2004;4(1):35-45.

62. Zawieja SD, et al. Differences in L-type $\mathrm{Ca}(2+)$ channel activity partially underlie the regional dichotomy in pumping behavior by murine peripheral and visceral lymphatic vessels. Am JPhysiol Heart Circ Physiol. 2018;314(5):H991-H1010.

63. Bouvree K, et al. Semaphorin3A, Neuropilin-1, and PlexinA1 are required for lymphatic valve formation. Circ Res. 2012;111(4):437-445.

64. Jurisic G, et al. An unexpected role of semaphorin3a-neuropilin-1 signaling in lymphatic vessel maturation and valve formation. Circ Res. 2012;111(4):426-436.

65. Beard RS Jr, et al. Non-muscle Mlck is required for beta-catenin- and FoxO1-dependent downregulation of Cldn 5 in IL-1beta-mediated barrier dysfunction in brain endothelial cells. J Cell Sci. 2014;127(Pt 8):1840-1853.

66. Taddei A, et al. Endothelial adherens junction control tight junctions by VE-cadherin-mediated upregulation of claudin-5. Nat Cell Biol. 2008;10(8):923-934.

67. Geng X, et al. Multiple mouse models of primary lymphedema exhibit distinct defects in lymphovenous valve development. Dev Biol. 2016;409(1):218-233.

68. Castorena-Gonzalez JA, et al. Simplified method to quantify valve back-leak uncovers severe mesenteric lymphatic valve dysfunction in mice deficient in connexins 43 and 37. JPhysiol. 2020;598(12):2297-2310.

69. Levet S, et al. Bone morphogenetic protein 9 (BMP9) controls lymphatic vessel maturation and valve formation. Blood. 2013;122(4):598-607.

70. Zhang G, et al. EphB4 forward signalling regulates lymphatic valve development. Nat Commun. 2015;6:6625.

71. Birkenkamp KU, Coffer PJ. Regulation of cell survival and proliferation by the FOXO (Forkhead box, class $\mathrm{O}$ ) subfamily of Forkhead transcription factors. Biochem Soc Trans. 2003;31(pt 1):292-297.

72. Zhang X, et al. Akt, FoxO, and regulation of apoptosis. Biochim Biophys Acta. 2011;1813(11):1978-1986.

73. Castrillon DH, et al. Suppression of ovarian follicle activation in mice by the transcription factor Foxo3a. Science. 2003;301(5630):215-218.

74. Castorena-Gonzalez JA, et al. Methods for assessing the contractile function of mouse lymphatic vessels ex vivo. Methods Mol Biol. 2018;1846:229-248. 